\title{
Warm ISM in the Sagittarius A Complex
}

\section{Mid-J CO, atomic carbon, ionized atomic carbon, and ionized nitrogen sub-mm/FIR line observations with the Herschel-HIFI and NANTEN2/SMART telescopes ${ }^{\star}, \star \star$}

\author{
P. García ${ }^{1}$, R. Simon ${ }^{1}$, J. Stutzki ${ }^{1}$, R. Güsten ${ }^{2}$, M. A. Requena-Torres ${ }^{2}$, and R. Higgins ${ }^{1}$ \\ ${ }^{1}$ I. Physikalisches Institut der Universität zu Köln, 50937 Cologne, Germany \\ e-mail: pablo@ph1.uni-koeln.de \\ 2 Max-Planck-Institut für Radioastronomie, Auf dem Hügel 69, 53121 Bonn, Germany
}

Received 26 May 2015 / Accepted 22 September 2015

\section{ABSTRACT}

\begin{abstract}
Aims. We investigate the spatial and spectral distribution of the local standard of rest (LSR) velocity resolved submillimetre emission from the warm $(25-90 \mathrm{~K})$ gas in the Sgr A Complex, located in the Galactic centre.

Methods. We present large-scale submillimetre heterodyne observations towards the Sgr A Complex covering $\sim 300 \operatorname{arcmin}^{2}$. These data were obtained in the frame of the Herschel EXtraGALactic guaranteed time key program (HEXGAL) with the HerschelHIFI satellite and are complemented with submillimetre observations obtained with the NANTEN2/SMART telescope as part of the NANTEN2/SMART Central Nuclear Zone Survey. The observed species are $\mathrm{CO}(J=4-3)$ at $461.0 \mathrm{GHz}$ observed with the NANTEN2/SMART telescope, and $[\mathrm{CI}]{ }^{3} \mathrm{P}_{1}-{ }^{3} \mathrm{P}_{0}$ at $492.2 \mathrm{GHz},[\mathrm{CI}]{ }^{3} \mathrm{P}_{2}-{ }^{3} \mathrm{P}_{1}$ at $809.3 \mathrm{GHz},[\mathrm{NII}]{ }^{3} \mathrm{P}_{1}-{ }^{3} \mathrm{P}_{0}$ at $1461.1 \mathrm{GHz}$, and [CII ${ }^{2} \mathrm{P}_{3 / 2}-{ }^{2} \mathrm{P}_{1 / 2}$ at $1900.5 \mathrm{GHz}$ observed with the Herschel-HIFI satellite. The observations are presented in a $1 \mathrm{~km} \mathrm{~s}^{-1}$ spectral resolution and a spatial resolution ranging from 46 arcsec to 28 arcsec. The spectral coverage of the three lower frequency lines is $\pm 200 \mathrm{~km} \mathrm{~s}^{-1}$, while in the two high frequency lines, the upper LSR velocity limit is $+94 \mathrm{~km} \mathrm{~s}^{-1}$ and $+145 \mathrm{~km} \mathrm{~s}^{-1}$ for the [NII] and [CII] lines, respectively.

Results. The spatial distribution of the emission in all lines is very widespread. The bulk of the carbon monoxide emission is found towards Galactic latitudes below the Galactic plane, and all the known molecular clouds are identified. Both neutral atomic carbon lines have their brightest emission associated with the $+50 \mathrm{~km} \mathrm{~s}^{-1}$ cloud. Their spatial distribution at this LSR velocity describes a crescent-shape structure, which is probably the result of interaction with the energetic event (one or several supernovae explosions) that gave origin to the non-thermal Sgr A-East source. The [CII] and [NII] emissions have most of their flux associated with the thermal arched-filaments and the $\mathrm{H}$ region and bright spots in [CII] emission towards the central nuclear disk (CND) are detected. Warm Gas at very high $\left(\left|V_{\mathrm{lsr}}\right|>100 \mathrm{~km} \mathrm{~s}^{-1}\right)$ LSR velocities is also detected towards the line of sight to the Sgr A Complex, and it is most probably located outside the region, in the $\mathrm{X}_{1}$ orbits.
\end{abstract}

Key words. ISM: atoms - ISM: molecules - ISM: clouds - Galaxy: center

\section{Introduction}

The Galactic centre (GC), located at $8.34 \pm 0.16 \mathrm{kpc}$ (Reid et al. 2014) from the Sun in the Sagittarius (Sgr) constellation, is one of the most remarkable places in the Milky Way; it harbours an overwhelming variety of astronomical sources under extraordinary physical conditions. The region between $l=-1.5$ to 3.5 and $b= \pm 0.75$, also known as the Nuclear Bulge (Mezger et al. 1996), harbours five giant molecular clouds (GMCs): Sgr A, Sgr B, Sgr C, Sgr D, and Bania's Clump 2 (Oka et al. 1998; Bania 1980; Stark \& Bania 1986). The densest GMCs within this region are located in the so-called central molecular zone (CMZ) extending from $l=-1.0$ to +1.5 and containing all GMCs in the Sgr A region.

\footnotetext{
* All data cubes presented in the paper (FITS files) are only available at the CDS via anonymous ftp to cdsarc.u-strasbg.fr $(130.79 .128 .5)$ or via http://cdsarc.u-strasbg.fr/viz-bin/qcat?J/A+A/588/A131 $\star \star$ Channel maps are availalble at https: //www . astro . uni-koeln.de/online-data/Garcia_P_SgrA_AA588/
}

The CMZ has been observed in almost the entire electromagnetic spectrum, ranging from centimetre wavelengths (Yusef-Zadeh et al. 1984; Law et al. 2008), millimetre wavelengths in a wide variety of molecules (Nagayama et al. 2007; Ott et al. 2014; Jones et al. 2013, 2012; Bally et al. 1987; Oka et al. 1998, 2012, 2007; Sawada et al. 2001), atomic, molecular, and dust observations in the submillimetre (sub-mm) range (Martin et al. 2004; Tanaka et al. 2011; Pierce-Price et al. 2000; Schuller et al. 2009; Bally et al. 2010), far- and mid-infrared observations accessible only from space (Molinari et al. 2011; Stolovy et al. 2006), optical imaging (Figer et al. 2004), and in the high energy range from a few tenths of $\mathrm{keV}$ up to $\mathrm{TeV}$ (Watson et al. 1981; Sidoli et al. 2001; Hunter et al. 1997; Aharonian et al. 2006) among others, and at a wide range of spectral and spatial resolutions. This large amount of astronomical data has revealed the complex kinematics and extreme physical conditions in the Nuclear Bulge (see Mezger et al. 1996 for a detailed review).

Among all GMCs in the CMZ, the Sgr A Complex is the one containing the dynamical centre of the Milky Way. The 
bolometric luminosity of the central parsec in the GC $\left(\sim 10^{8} L_{\odot}\right)$ accounts for $\sim 0.3 \%$ of the bolometric luminosity of the Galaxy. Given the low luminosity of the GC, it is thought that the Milky Way would resemble a weak Seyfert Galaxy as seen from a distance of $700 \mathrm{kpc}$, with an angular resolution of 0.05" (Mezger et al. 1996). Understanding the physical conditions and dynamics of the Sgr A Complex is crucial to understanding unresolved CMZs in other Galaxies.

The Sgr A region, extending $\sim 100 \mathrm{pc}$ around $\mathrm{Sgr} \mathrm{A}^{\star}$, contains a rich diversity of prominent astronomical sources confined in a small volume, whose emission can be detected across the entire spectral domain. Some of these sources are the non-thermal shell Sgr A-East and the H II region Sgr A-West (Yusef-Zadeh \& Morris 1987a); the central nuclear disk (CND; Requena-Torres et al. 2012); the radio arc and several non-thermal filaments (NTFs) running perpendicular to the Galactic plane and possibly associated with the poloidal structure of the magnet-field in this region (Yusef-Zadeh \& Morris 1987b; Lang et al. 1999b, 2010); the Thermal Arched Filaments (Serabyn \& Guesten 1987; Lang et al. 2002); the H Region containing several H II regions (Lang et al. 2001; Yusef-Zadeh \& Morris 1987a; Zhao et al. 1993); the Sickle and Pistol star (Yusef-Zadeh \& Morris 1987b; Timmermann et al. 1996; Lang et al. 1997); the three massive star clusters Quintuplet, Arches, and the Nuclear Cluster (Cotera et al. 1996; Lang et al. 1999a; Chatzopoulos et al. 2015); several molecular clouds (MCs) such as the $-30 \mathrm{~km} \mathrm{~s}^{-1},+20 \mathrm{~km} \mathrm{~s}^{-1}$, and $+50 \mathrm{~km} \mathrm{~s}^{-1}$ clouds (Güsten et al. 1981; Serabyn \& Guesten 1987; Zhao et al. 1993); water and methanol masers (Caswell et al. 2010, 2011); X-ray point-like sources such as 1E-1743.12843B (Porquet et al. 2003); and high velocity compact clumps (HVCCs) (Oka et al. 2008). Along the same line of sight (1.o.s.) but outside this region are found gas at high local standard of rest (LSR) velocities associated with $\mathrm{X}_{1}$ orbits and absorption features at LSR velocities $-55 \mathrm{~km} \mathrm{~s}^{-1},-30 \mathrm{~km} \mathrm{~s}^{-1}$, and $-5 \mathrm{~km} \mathrm{~s}^{-1}$ associated with the loci of the $3 \mathrm{kpc}, 4.5 \mathrm{kpc}$, and local arm (Oka et al. 1998; Jones et al. 2012; Luna et al., in prep.).

In the sub-mm/far-infrared (FIR) range (100 GHz-10 THz), great effort has been made to understand the physics behind the Sgr A Complex and the numerous astronomical sources within it. On the one hand, previous heterodyne observations of this region, ranging from a few targeted positions with moderate spectral resolution to large-scale observations with moderate spatial resolution, have largely contributed to understanding the kinematics and excitation mechanisms of the warm gas in the GC (Genzel et al. 1990; Poglitsch et al. 1991; Mizutani et al. 1994; Martin et al. 2004). More recently, small-scale observations with high spatial and spectral resolutions have become available for the shorter wavelengths in this spectral domain with the Stratospheric Observatory for Infrared Astronomy (SOFIA) as shown in the observations of the CND by Requena-Torres et al. (2012). Additionally, several high resolution continuum observations covering the whole CMZ have been crucial to estimating volume densities and temperatures and to characterizing the spatial morphology of the warm gas in this region (Mezger et al. 1989; Pierce-Price et al. 2000; Bally et al. 2010; Molinari et al. 2011).

Observation with ground-based telescopes in this frequency domain are particularly difficult given the poor atmospheric transmission. In the present work we present observations of the entire Sgr A Complex at high angular $\left(\leq 46^{\prime \prime}\right)$ and spectral $\left(1 \mathrm{~km} \mathrm{~s}^{-1}\right)$ resolution in most of the main cooling lines (except for the two [OI] transitions at $146 \mu \mathrm{m}$ and $63 \mu \mathrm{m}$ ) in the sub-mm/FIR regime of the ISM $(\mathrm{CO}(4-3),[\mathrm{CI}](1-0)$, $[\mathrm{CI}](2-1)$, and $[\mathrm{CII}])$ and $\mathrm{H}$ II regions ([NII]) obtained with the
Herschel-HIFI satellite and the NANTEN2/SMART telescope. In Sect. 2, the data acquisition and data reduction process are described. In Sect. 3 the main sources detected in the observations are discussed. In Sect. 4 we summarize the work described in the previous sections and outline the future work in terms of the excitation analysis (PDRs vs XDRs, LVG analysis, etc.) of the gas associated with the variety of sources in our observations which are under very different physical conditions.

\section{Data reduction}

In the following, we describe the data acquisition and data reduction process of the submillimetre lines observed with the Sub-Mm Array Receiver for Two frequencies (SMART) at the NANTEN2 telescope, and the Heterodyne Instrument for the Far-Infrared (HIFI) on board the Herschel satellite. We conclude with a summary of the main parameters characterizing our observations.

\subsection{Herschel-HIFI [CI]1-0, [CI]2-1, [NII], and [CII] observations}

With the Herschel-HIFI instrument, large-scale mapping of four submillimetre lines towards the Sgr A Complex was carried out in the frame of the Herschel Guaranteed Time HEXGAL Key Program (P.I. R. Güsten, MPIfR): [CI ${ }^{3} \mathrm{P}_{1}-{ }^{3} \mathrm{P}_{0}$ (OBS IDs 1342205504 and 1342205505 ), $[\mathrm{CI}]{ }^{3} \mathrm{P}_{2}-{ }^{3} \mathrm{P}_{1}$ (OBS IDs 1342204733 and 1342204734), [NII ${ }^{3} \mathrm{P}_{1}-{ }^{3} \mathrm{P}_{0}$ (OBS IDs 1342205892, 1342205893，1342205894, 1342206599, 1342216664, and 1342216665), and $[\mathrm{CII}]{ }^{2} \mathrm{P}_{3 / 2}-{ }^{2} \mathrm{P}_{1 / 2}$ (OBS ID $1342206647,1342206648,1342206649,1342206650$, 1342206651, and 1342206652). The HIFI Instrument is a double sideband (DSB) single pixel receiver, with a total of seven mixer bands, ranging in frequency continuously from $480 \mathrm{GHz}$ to $1250 \mathrm{GHz}$ and from 1410 up to $1910 \mathrm{GHz}$. The mixing devices are SIS (superconductor-insulator-superconductor) mixers for bands 1 to 5 (4 GHz bandwidth) and HEB (hot-electron bolometer) mixers for bands 6 and 7 (2.4 GHz bandwidth). Each band is in turn divided into two sub-bands, labelled with the letters "a" and " $b$ ", each one associated with a different local oscillator (LO) chain. The backends used were two wide-band acousto-optical spectrometers (WBS), one for each measured vertical $(\mathrm{V})$ and horizontal $(\mathrm{H})$ polarization. The spectral resolution of the WBS is $1.1 \mathrm{MHz}$ and possesses a total bandwidth of $4 \mathrm{GHz}$ (de Graauw et al. 2010).

The bands $1 \mathrm{a}, 3 \mathrm{a}, 6 \mathrm{a}$, and $7 \mathrm{~b}$, correspond to the $[\mathrm{CI}](1-0)$, $[\mathrm{CI}](2-1),[\mathrm{NII}]$, and $[\mathrm{CII}]$ observed atomic and ionic transitions, respectively. The pointing accuracy of the telescope was estimated to be $\sim 2^{\prime \prime}$ ' and the calibration error in the measured absolute antenna temperatures ranges from $15 \%$ in band 1a up to $25 \%$ in band $7 \mathrm{~b}$ (Roelfsema et al. 2012).

\subsubsection{Observing strategy}

The observations were carried out in the equatorial coordinates reference frame and two observing modes were used for different frequencies: the On-The-Fly (OTF) map with positionswitch reference observing mode for the $[\mathrm{CI}](1-0)$ line, and the OTF map with load chop and position-switch reference observing mode for the $[\mathrm{CI}](2-1),[\mathrm{NII}]$, and [CII] lines. The choice between them is mainly driven by the Allan stability time (Allan 1966; Ossenkopf 2008) of the receiver at a given frequency (Roelfsema et al. 2012). 

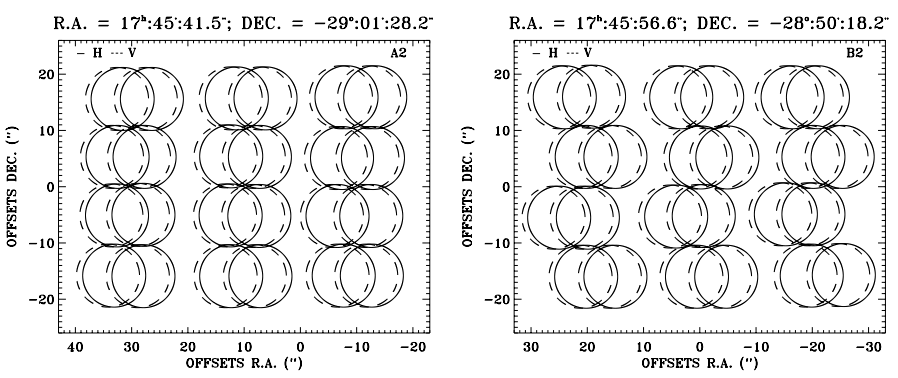

Fig. 1. Examples of the $[\mathrm{CII}]$ observed grid in two different subregions of the entire observed map. The beam size at this frequency is represented by the circle's size. The H polarization (solid line) and $\mathrm{V}$ polarization (dashed line) spectra are shown. The slight misalignment between the beams is $-1.0^{\prime \prime}$ in the $Y$ and $0.0^{\prime \prime}$ in the $Z$ focal plane coordinates of the HIFI instrument. The OTF map with load chop and position-switch reference observing mode used for the high frequency lines undersampled the observed maps.

The OTF map with load chop and position-switch reference is very efficient in producing integration times on source that are shorter than the Allan stability times of the high frequency lines; however, one disadvantage is that it yields an irregular sampling pattern of the observed area leading to observed maps that are undersampled in the scanning direction given the beam size at the observed frequency. An example of this is shown in Fig. 1 for the $[\mathrm{CII}]$ line. The figure shows the sampling pattern of two different subregions observed. The absolute equatorial coordinates of the central position $(0,0)$ of each map are given at the top of each panel. The beam size at this frequency is $11.2^{\prime \prime}$ and is represented by the plotted circles. Also, both polarizations, $\mathrm{H}$ (solid circle) and V (dashed circle), are shown. The beams of the two polarizations are shifted as a result of a small misalignment due to the construction process, measured to be $-1.0^{\prime \prime}$ in the $Y$ and $0.0^{\prime \prime}$ in the $Z$ focal plane coordinates of the receiver (Roelfsema et al. 2012). The angular separation between two consecutive spectra is $5.1^{\prime \prime}$, while the separation for the spectra immediately before and after the chop to the internal load is $15.3^{\prime \prime}$, larger than the beam size at this frequency. The separation of the OTF lines in the declination is $10.5^{\prime \prime}$, almost the size of the beam. From the figure it can be seen that the original measured map is undersampled in the scanning direction (right ascension). In the declination direction, the map is beam sampled by design. The same sampling pattern is present in the $[\mathrm{NII}]$ and $[\mathrm{CI}](2-1)$ lines observations though the undersampled area is lower given the larger beam sizes.

\subsubsection{HIFI data reduction process}

The data reduction software for all instruments on board the Herschel satellite is the Herschel interactive processing environment (HIPE; Ott 2010). For each instrument a data processing pipeline processes the raw data counts into frequency and intensity calibrated data. This process is broken into a number of discrete processing steps unique to each observing mode (Roelfsema et al. 2012). The output from the pipeline at different steps can be examined, which is especially useful in dealing with situations in which the final output appears problematic. For this work, the level2 data were taken. The first processing step after the standard level 2 pipeline is the "Stitch" Task (full name herschel.ia.toolbox.spectrum.StitchSpectrumTask). This task takes the overlapping region among the sub-bands, calculates the average values in that range separately, brings

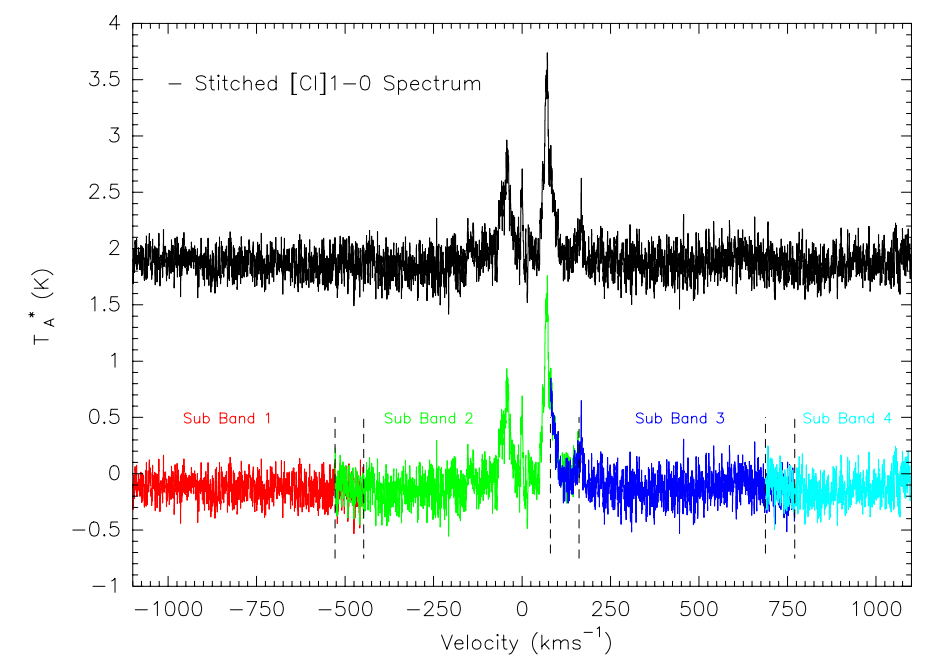

Fig. 2. $[\mathrm{CI}](1-0)$ spectrum showing the $1 \mathrm{GHz}$ WBS sub-bands before (colours) and after (black) the stitching procedure. The dashed vertical lines encompass the overlapping spectral region between consecutive sub-bands. After the stitching procedure, the full spectrum is used in the data reduction process. The displacement in the continuum level towards higher antenna temperatures of the end product spectrum is artificially introduced for display purposes

them to the same continuum level, and glues the sub-bands together. An example of this procedure is shown in Fig. 2 for the observed $[\mathrm{CI}](1-0)$ line. The colours represent the four different $1 \mathrm{GHz}$ WBS sub-bands. The velocity resolution at this frequency is $0.305 \mathrm{~km} \mathrm{~s}^{-1}$. The overlapping regions between sub-bands are enclosed between vertical dashed lines. The results of the stitching procedure are shown in the full spectrum (black line) on the same plot.

The raw spectra were exported to GILDAS compatible FITS (Flexible Image Transport System) files using the "Hiclass" task in HIPE to further continue the data reduction process in the CLASS module of GILDAS software ${ }^{1}$. In the case of the Sgr A Complex the emission lines cover a very wide LSR velocity range, between $-200 \mathrm{~km} \mathrm{~s}^{-1}$ and $+200 \mathrm{~km} \mathrm{~s}^{-1}$. Since spectral resolution of the HIFI receiver is very high, we resampled all spectra to $1 \mathrm{~km} \mathrm{~s}^{-1}$ spectral resolution. This ensures that emission lines are spectrally resolved, at the same time increasing the signal-to-noise ratio. For the baseline subtraction, polynomials of orders 1-3 were used. Spectra that have anomalies in their baselines can usually be filtered out by setting a threshold to the rms temperature $\left(T_{A, \mathrm{rms}}^{*}\right)$ tolerance, but given the excellent stability of the HIFI receiver in bands $1 \mathrm{a}$ and $3 \mathrm{a}$, none of the spectra were discarded.

\subsubsection{Special cases}

The baseline fitting procedure for the [NII] and [CII] lines in bands $6 \mathrm{a}$ and $7 \mathrm{~b}$, respectively, requires a slightly different approach. The HEB mixers in bands $6 \mathrm{a}$ and $7 \mathrm{~b}$ show a reflection in the electrical amplification chain, which leads to distortion of the baselines in the spectra measured at these frequencies (Higgins 2011).

In band $6 a$, the spectra show a baseline oscillation at one edge of the bandpass, between $+94 \mathrm{~km} \mathrm{~s}^{-1}$ and $+170 \mathrm{~km} \mathrm{~s}^{-1}$, in the $\mathrm{V}$ and the $\mathrm{H}$ polarizations. This perturbation is shown in

1 https://wWw.iram.fr/IRAMFR/GILDAS/ 


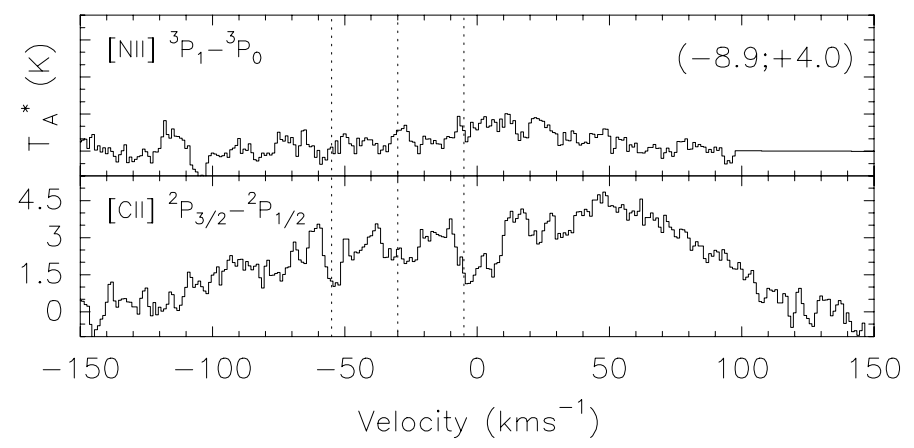

Fig. 3. [NII] and [CII]spectra at position $\Delta \alpha(J 2000)=-8.9^{\prime \prime}$, $\Delta \delta(\mathrm{J} 2000)=+4.0^{\prime \prime}$ after the filtering process explained in Sect. 2.1.3 and after the final data cube was created. The straight line at LSR velocities $>+94 \mathrm{~km} \mathrm{~s}^{-1}$ in the [NII] spectrum is the result of the method utilized in removing the baseline distortion (see text).

Fig. A.1 (see Appendix A) as a red line in the same LSR velocity range. The spectrum shown is the average spectrum of the $\mathrm{H}$ polarization over the whole data set. Unfortunately, given the very wide velocity dispersion of the line, this perturbation affects regions of the bandpass where emission is present. The origin of this anomaly is related to a combination of problems: the diplexer coupling at this frequency, the electrical standing waves in the HEB mixers, and the IF (intermediate frequency) frequency dependence sensitivity of the HEB mixers that are more sensitive to lower IF frequencies (Higgins 2011). In order to minimize the impact of this oscillation on the final data set, the average $T_{A}^{*}$ value between $+94 \mathrm{~km} \mathrm{~s}^{-1}$ and $+170 \mathrm{~km} \mathrm{~s}^{-1}$ was calculated and assigned to the measured antenna temperature before subtracting the baseline from the spectra. The result of this procedure is shown as a black dashed line in the same figure. We investigated the effect of a polynomial of order 0 and 1 subtracted from the spectra and the best results were obtained with the latter. In this way, the usable LSR velocity range of this data set is from $-200 \mathrm{~km} \mathrm{~s}^{-1}$ to $+94 \mathrm{~km} \mathrm{~s}^{-1}$ only.

In band $7 \mathrm{~b}$, the $\mathrm{V}$ polarization HEB mixer suffers from a too high LO power, which yields an impedance mismatch between the mixer and the first low noise amplifier (LNA) as was shown by Higgins (2011). Since both polarizations share the same LO chain, optimizing the LO power for one of the polarizations unavoidably causes the LO power of the other polarization to be either too low (in the case of the $\mathrm{H}$ polarization HEB mixer) or too high (V polarization HEB mixer). In the Herschel-HIFI observation scheme, it was decided to operate the $\mathrm{H}$ polarization HEB mixer at the optimum level, namely, at a mixer current of $0.045 \mathrm{~mA}$, and the $\mathrm{V}$ polarization mixer current was left to follow (Higgins 2011). As a consequence, some of the V polarization spectra suffer from large distortions of their baselines. An example of the $\mathrm{V}$ polarization spectra is shown in Fig. A.2 (see Appendix A). The figure shows the average spectrum of one OTF line. We set a $T_{A, \text { rms }}^{*}=2.3 \mathrm{~K}$ threshold to filter out the bad spectra from the $\mathrm{V}$ polarization data set. For comparison with Figs. A.1 and A.2, single [NII] and [CII] spectra at position $\Delta \alpha(\mathrm{J} 2000)=-8.9^{\prime \prime}, \Delta \delta(\mathrm{J} 2000)=+4.0^{\prime \prime}$ (the closest position to the centre of our maps) after the final data cubes were created are shown in Fig. 3. The straight line at LSR velocities $>+94 \mathrm{~km} \mathrm{~s}^{-1}$ in the [NII] spectrum is the result of the interpolation process applied to that data set.

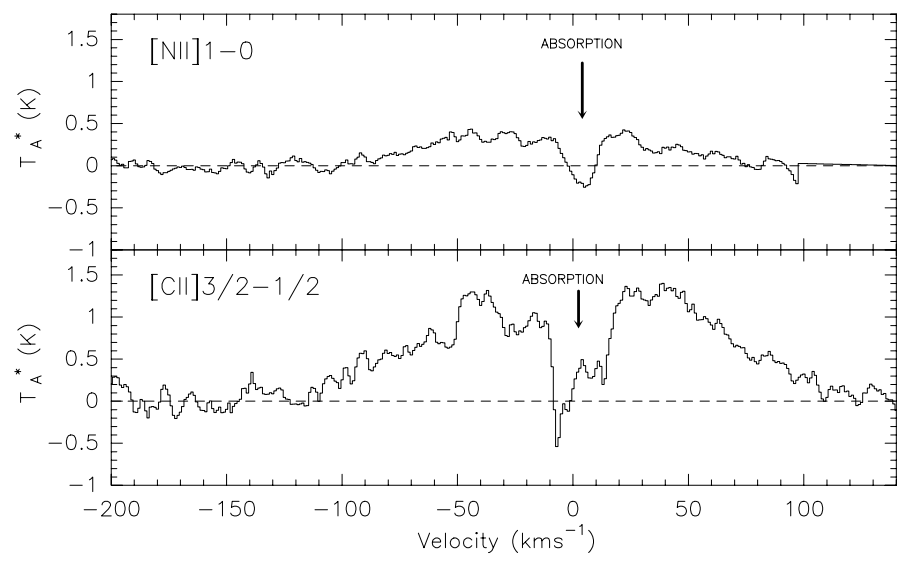

Fig. 4. Average spectra of the Herschel-HIFI [NII] and [CII] observations. The arrows show the positions of the absorption features present in the data due to the contamination of the reference position (SKY) with line emission.

\subsubsection{Emission contamination in the reference position}

The reference position of the Herschel-HIFI observations is located at $\alpha(\mathrm{J} 2000)=17^{\mathrm{h}} 44^{\prime} 33^{\prime \prime}, \delta(\mathrm{J} 2000)=-28^{\circ} 52^{\prime} 08^{\prime \prime}$ in equatorial coordinates for all four lines observed. The selection of this position was based on a compromise between the absence of polycyclic aromatic hydrocarbon emission detected by the SPIRIT III infrared telescope at $8.3 \mu \mathrm{m}$ as part of the Midcourse Space Experiment (MSX) Galactic Plane Survey, and a reference position not far from the astronomical target. Unfortunately, all four lines observed with the Herschel-HIFI receiver suffer from emission contamination in the reference position. Figure 4 shows the average spectrum of the $[\mathrm{NII}]$ and $[\mathrm{CII}]$ lines over the entire observed region. A massive absorption feature can be seen in both spectra, around $0 \mathrm{~km} \mathrm{~s}^{-1}$, going well below the $0 \mathrm{~K}$ antenna temperature in the baseline subtracted spectra.

Absorption features are expected to be found when observing towards the Galactic Centre due to the presence of foreground material (such as spiral arms) along the l.o.s. (Linke et al. 1981). In order to identify whether the absorption features seen in Fig. 4 are the result of a contaminated reference position or of the foreground absorbing material, a calibrated spectrum of the reference position is needed in order to assess whether there is emission there. The most common way to recover the emission of a contaminated reference position is to select an area of the observed region that contains no emission, usually towards the edges of the maps. From the selected spectra, an average spectrum with lower noise temperature than the rest of the map can be created. After that, the observed absorption features are inverted and added to all the spectra in the map. Unfortunately, this approach cannot be used in the case of the Herschel-HIFI observations. The emission distribution is very extended in all lines, so there is no position free of emission in the map. Therefore, a different approach must be used.

Given the observing mode used in some of the HerschelHIFI observed data sets, it is possible to reconstruct the reference position spectrum for each of the OTF lines observed. The calibration of the measured reference position is possible for all lines observed in the OTF Map With Load Chop And PositionSwitch Reference observing mode such as [CI] $(2-1)$, [NII], and [CII]. This observing mode chops to internal loads immediately before taking a spectrum. The chop to internal loads is used to determine the bandpass of the receiver and is done for the on source position as well as for the reference position. In this way, 
the standard data reduction pipeline of the Herschel-HIFI observations can be applied to convert the measured counts at the reference position into fully calibrated reference spectra in the $T_{A}^{*}$ temperature scale. Nonetheless, there are telescope related artefacts, such as standing waves produced by the difference in light path when chopping to the internals loads, that are not removed from the SKY spectra during the standard calibration process. In this sense, the reference spectra are calibrated properly in temperature scale, but contain standing waves, whose shapes depend on the utilized spectral band and have to be removed in order to recover the line emission. This procedure is explained in Sect. 2.1.5. The corresponding amplitudes of these standing waves are of the order of $\sim 0.5 \mathrm{~K}$ for the $[\mathrm{CII}]$ and $[\mathrm{NII}]$ lines, and $\sim 1 \mathrm{~K}$ for the $[\mathrm{CI}](2-1)$ line.

\subsubsection{Model of the reference position emission}

In order to account for the subtracted reference emission from the data, we modelled the emission at the reference position and added it back to each individual spectrum in a series of steps: (a) we calibrated each observed reference position producing a calibrated spectrum in $T_{A}^{*}$ temperature scale; (b) we fit a 0 -order polynomial to the spectra and selected only the ones with the lowest noise. Thus, an average spectrum with much lower noise than the spectra in the map is created. The selected spectra used to create the average spectrum are $\sim 100$ for the $[\mathrm{CI}](2-1)$ line and around $\sim 200$ for the [NII] and [CII] lines; (c) we removed only well-defined standing waves identified in a Fourier transform of the average spectrum. This was possible because all standing waves have a consistent structure in all spectra, so adding them increases the signal-to-noise ratio but preserves their structure. Well-defined standing waves appear as very narrow spikes in the time domain, so it is, in principle, possible to remove them by interpolating the intensity just before and after the spike without affecting the true emission structure of the spectrum. Ideally, after doing this, the velocity structure of the line should be compared with other observations - obtained by independent observations - of similar tracers at the same position in order to avoid introducing artificial structures to the data (see below); (d) a low-order baseline was fitted and subtracted from the resulting average spectrum where the emission was already clearly visible; (e) we modelled the emission by fitting up to five independent Gaussians to the average spectrum creating an emission model that was added to each spectrum in the observed map. This procedure effectively recovers the emission contamination in the reference position and avoids adding extra noise to the data apart from the uncertainties in the fitted Gaussian parameters.

We checked that no artificial emission was created during the removal of the standing waves by trying different interpolations and checking the variations in the results. Only when the standing wave was very well defined as a narrow intensity peak in the time domain and its radial velocity coincided with the radial velocity corresponding to the absorption features in the average spectrum of the corresponding line (as shown in Fig. 4) was the resulting spectrum used to create the emission model. The difference in the measured positions of the reference spectra is of the order of the absolute pointing error (APE) of the telescope $\sim 2^{\prime \prime}$ (Roelfsema et al. 2012), so it is assumed that all reference spectra were measured at the same position on the sky.

The results of the procedure are shown in Fig. 5. The black line in panels $(a-c)$ represent the average reference spectrum for the [NII], [CII], and [CI] (2-1) lines, respectively (see Sect. 2.1.6 for $[\mathrm{CI}](1-0))$. The noise in each spectrum is $\sigma=0.196 \mathrm{~K}$ for

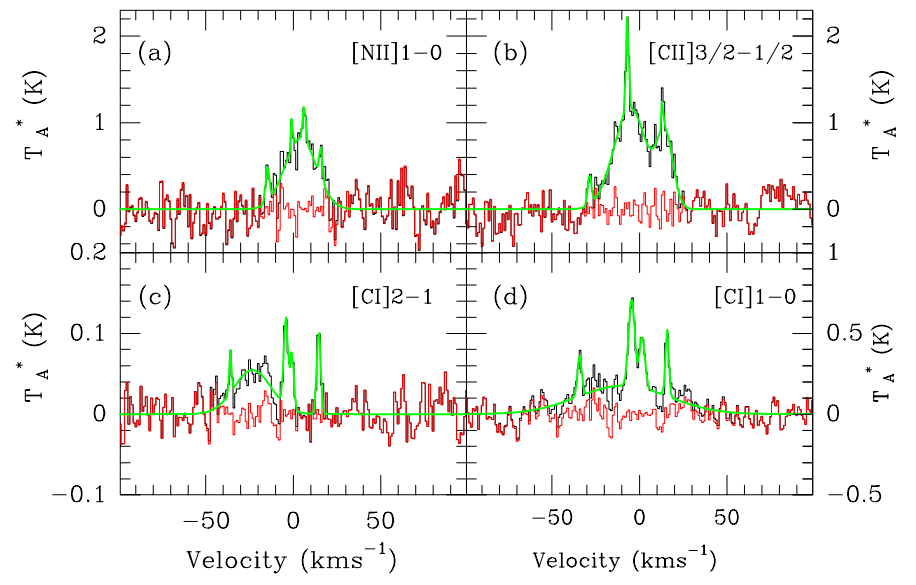

Fig. 5. Panels a), b), c), and d): reference position calibrated average spectrum (black), the fitted emission model (green), and the residual after the subtraction of the fitted model from the reference position emission (red), for the $[\mathrm{NII}],[\mathrm{CII}],[\mathrm{CI}](2-1)$, and $[\mathrm{CI}](1-0)$ observations, respectively.

the [NII] line, $\sigma=0.173 \mathrm{~K}$ for the [CII] line, and $\sigma=0.022$ $\mathrm{K}$ for the $[\mathrm{CI}](2-1)$ line. In all three cases, the noise of the average reference spectrum is more than 10 times lower than the typical noise in the map. The green line is the emission model constructed from the fitting of up to five independent Gaussians to the observed emission. We reproduce all observed emission above a $\sim 2.5 \sigma$ noise level. The quality of the model in reproducing the emission can be assessed from the residual spectra (red line) showing the difference between the observed spectrum and the fitted model across the whole velocity range. All three models reproduce the emission contained in the average reference spectrum very well. The $[\mathrm{CI}](1-0)$ profile in panel $\mathrm{d}$, obtained by independent measurements of the reference position (with $\sigma=0.064 \mathrm{~K}$, see Sect. 2.1.6), shows almost exactly the same velocity structure as the $[\mathrm{CI}](2-1)$ profile in panel $\mathrm{c}$, and is also similar to the $[\mathrm{CII}]$ profile in panel $\mathrm{b}$; this strongly supports our approach to determining the emission structure at the reference position.

We also compared the average spectrum before and after adding the emission model to each individual spectrum (see Fig. 6). From top to bottom, the models of the [NII], [CII], and $[\mathrm{CI}](2-1)$ emission in the reference position recover all the most important absorption features shown in Fig. 4. In the case of the [NII] line, the emission in the reference position is much stronger than the average emission over the map, so the model shape stands out more clearly than in the other lines, as seen in the intensity peak around $+5 \mathrm{~km} \mathrm{~s}^{-1}$. After the recovery of the emission at the reference position has been added back to the data sets, we assume that only real physical absorption features along the l.o.s. are to be found.

\subsection{6. $[\mathrm{Cl}](1-0)$ reference emission}

In the case of the $[\mathrm{CI}](1-0)$ line, the OTF Map With PositionSwitch Reference observing mode was used to carry out the observations so we adopted a different approach to check for emission contamination in the reference position. In this observing mode, given the stability of the receiver, no chop to the internal loads is needed before each ON read out. Instead, a load measurement was done after each OTF line. In principle, it is possible to obtain as many fully calibrated reference spectra as there are OTF lines by using the load measurement 
Table 1. Summary of data sets after data reduction process.

\begin{tabular}{|c|c|c|c|c|c|c|c|c|c|c|c|}
\hline Telescope & Line & $\begin{array}{c}v_{\mathrm{obs}} \\
(\mathrm{GHz})\end{array}$ & $\begin{array}{c}\Theta_{\mathrm{FWHM}} \\
\left({ }^{\prime \prime}\right)\end{array}$ & $\begin{array}{c}\Theta \\
\left({ }^{\prime \prime}\right) \\
\end{array}$ & $F_{\text {eff }}$ & $B_{\text {eff }}$ & $\begin{array}{c}T_{A, \mathrm{rms}}^{*} \\
(\mathrm{~K})\end{array}$ & $\begin{array}{c}\Delta V_{\text {res }} \\
\left(\mathrm{km} \mathrm{s}^{-1}\right)\end{array}$ & $\begin{array}{c}\Delta V_{f} \\
\left(\mathrm{~km} \mathrm{~s}^{-1}\right)\end{array}$ & $\begin{array}{c}\Delta V_{\text {range }} \\
\left(\mathrm{km} \mathrm{s}^{-1}\right)\end{array}$ & $\begin{array}{l}E_{\mathrm{u}} \\
(\mathrm{K}) \\
\end{array}$ \\
\hline NANTEN2/SMART & $\mathrm{CO}(J=4-3)$ & 461.0 & 37.4 & 40.0 & 0.86 & 0.50 & 0.14 & $0.677^{a} / 0.050^{b}$ & 1.0 & -200 to +200 & 55 \\
\hline Herschel-HIFI & {$[\mathrm{CI}]{ }^{3} \mathrm{P}_{1}-{ }^{3} \mathrm{P}_{0}$} & 492.2 & 43.1 & 46.0 & 0.96 & 0.62 & 0.06 & 0.305 & 1.0 & -200 to +200 & 25 \\
\hline Herschel-HIFI & {$[\mathrm{CI}]{ }^{3} \mathrm{P}_{2}-{ }^{3} \mathrm{P}_{1}$} & 809.3 & 26.2 & 28.0 & 0.96 & 0.63 & 0.13 & 0.185 & 1.0 & -200 to +200 & 60 \\
\hline Herschel-HIFI & {$[\mathrm{NII}]{ }^{3} \mathrm{P}_{1}-{ }^{3} \mathrm{P}_{0}$} & 1461.1 & 14.5 & 46.0 & 0.96 & 0.58 & 0.28 & 0.103 & 1.0 & -200 to +94 & 70 \\
\hline Herschel-HIFI & {$[\mathrm{CII}]{ }^{2} \mathrm{P}_{3 / 2}-{ }^{2} \mathrm{P}_{1 / 2}$} & 1900.5 & 11.2 & 46.0 & 0.96 & 0.59 & 0.42 & 0.079 & 1.0 & -200 to +144 & 90 \\
\hline
\end{tabular}

Notes. ${ }^{(a)}$ For AOS backends. ${ }^{(b)}$ For XFFTS backends.

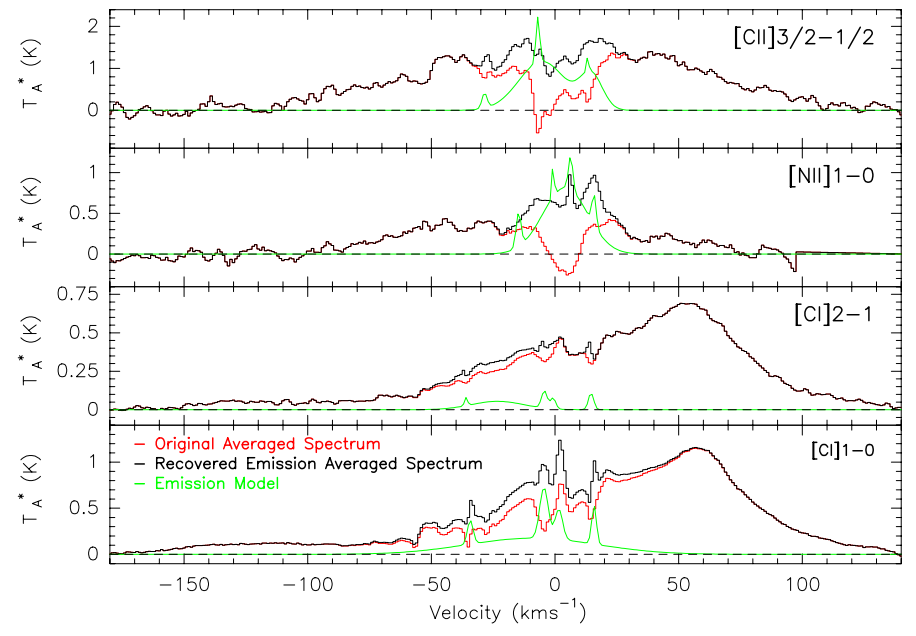

Fig. 6. From top to bottom, the $[\mathrm{CII}]$, $[\mathrm{NII}],[\mathrm{CI}](2-1)$, and $[\mathrm{CI}](1-0)$ average spectra before (red) and after (black) the addition of the reference position emission model (green) to each individual spectrum are shown. The absorption features due to the contamination of the reference position in all four lines are fully recovered.

and reference position integration closest in time. Nonetheless, this approach requires a major modification of the standard HIFI data reduction pipeline. More importantly, we chose a different method than the one mentioned in the previous section in order to have independent measurement of the $[\mathrm{CI}](1-0)$ profile at the reference position, so the quality of the method used for the other lines can be assessed (see previous section). We used the NANTEN2/SMART telescope to re-observe the Herschel-HIFI reference position against a reference position that was further away. We observed a $30^{\prime \prime} \times 30^{\prime \prime}$ map, centred at the reference position, with $10^{\prime \prime}$ spacing in both directions. Given the better spatial resolution of the $4 \mathrm{~m}$ NANTEN2/SMART telescope, we smoothed the map to a resolution equivalent to the beam size (FWHM) of the Herschel-HIFI telescope at this frequency (43.1"). The average spectrum of the map (Fig. 5, panel d) has a rms noise of $\sigma=0.064 \mathrm{~K}$ in the $T_{A}^{*}$ antenna temperature scale, which is lower than the rms noise of all Herschel-HIFI spectra measured at this frequency. The emission model (green line) reproduces the main features in the spectrum very well, and yields a residual spectrum (red line) consistent with pure noise.

In order to recover the emission subtracted from the ON spectra, we added the modelled emission channel by channel to the Herschel-HIFI [CI](1-0) spectra. We assume by this procedure that both telescopes measure the same antenna temperatures in the $T_{A}^{*}$ scale, an assumption that is fully supported by the analysis in Sect. 2.3.1. In Fig. 6, a comparison between the average spectrum, before and after recovering the emission subtracted from the on-source spectra, is shown. The remaining absorption features in the average spectrum are due to foreground material along the l.o.s.

After the baseline subtraction and OFF emission recovery processes were performed, all data sets were brought to positionvelocity data cubes using the Grenoble Graphic (GREG) software, also part of the GILDAS package. The spatial resolution of the $[\mathrm{CI}](1-0)$ and $[\mathrm{CI}](2-1)$ data cubes $\left(46^{\prime \prime}\right.$ and $28^{\prime \prime}$, respectively) is slightly lower $(\sim 6 \%)$ than the corresponding beam sizes of the observations (see Table 1). This is due to the Gaussian convolution kernel in GREG, which is one-third the size of the telescope beam width. For the case of the [NII] and [CII] lines, we set the convolution kernel size so the data match the $46^{\prime \prime}$ spatial resolution of the $[\mathrm{CI}](1-0)$ data cube, improving the signal-to-noise ratio of the observations in the processs. The spatial noise distribution of the Herschel-HIFI data sets is shown in the panels of Fig. 7, together with a histogram of the $T_{A, \text { rms }}^{*}$ values (upper panels). The Gaussian profiles fitted to the $T_{A, \mathrm{rms}}^{*}$ histograms are used only as a convenient approximation of the $T_{A, \text { rms }}^{*}$ true distribution to quantify the characteristic noise of each data set. A more complex chi-squared distribution would reproduce the $T_{A, \mathrm{rms}}^{*}$ distribution more accurately (Goldsmith et al. 2002), but such an analysis is outside the scope of the present work.

\subsection{NANTEN2/SMART CO(4-3) observations}

As part of the NANTEN2/SMART Central Molecular Zone Survey, the $\mathrm{CO}(4-3)$ line was observed towards the Sgr A Complex. Other submillimetre observations such as the $[\mathrm{CI}](1-0)$ line were carried out to obtain a cross-calibration between the Herschel-HIFI and NANTEN2/SMART observations as shown and discussed in Sect. 2.3.2. The SMART instrument (Graf et al. 2003) is a DBS $2 \times 8$ pixel heterodyne receiver operating at $460 \mathrm{GHz}$ and $810 \mathrm{GHz}$ frequencies simultaneously. The tunable range of the two frequencies spans from $435 \mathrm{GHz}$ to $495 \mathrm{GHz}$ for the $460 \mathrm{GHz}$ channels and from $795 \mathrm{GHz}$ to $880 \mathrm{GHz}$ for the $810 \mathrm{GHZ}$ channels, with an intermediate frequency $\left(v_{\mathrm{IF}}\right)$ of $4 \mathrm{GHz}$ and $1.5 \mathrm{GHz}$, respectively. The mixing devices are $4 \mathrm{GHz}$ bandwidth SIS mixers for both channels. During the data acquisition campaign, two different backends were used: Acousto Optical Spectrometers (AOSs; Horn et al. 1999) and eXtended bandwidth Fast Fourier Transform Spectrometers (XFFTSs; Klein et al. 2012). The AOSs were used from the beginning of the data acquisition campaign until August 2013. They have a $1 \mathrm{GHz}$ bandwidth with a $1.04 \mathrm{MHz}$ spectral resolution corresponding to $0.68 \mathrm{~km} \mathrm{~s}^{-1}$ at $460 \mathrm{GHz}$. About $75 \%$ of the data were observed using the AOSs as the backend before switching to the new XFFTSs. The XFFTSs were installed in September 2013. They posses a total bandwidth of $2.5 \mathrm{GHz}$ and a spectral resolution of $88.5 \mathrm{KHz}$, corresponding to $0.05 \mathrm{~km} \mathrm{~s}^{-1}$ at $460 \mathrm{GHz}$. With both backends, we 
P. García et al.: Warm ISM in the Sagittarius A Complex. I.
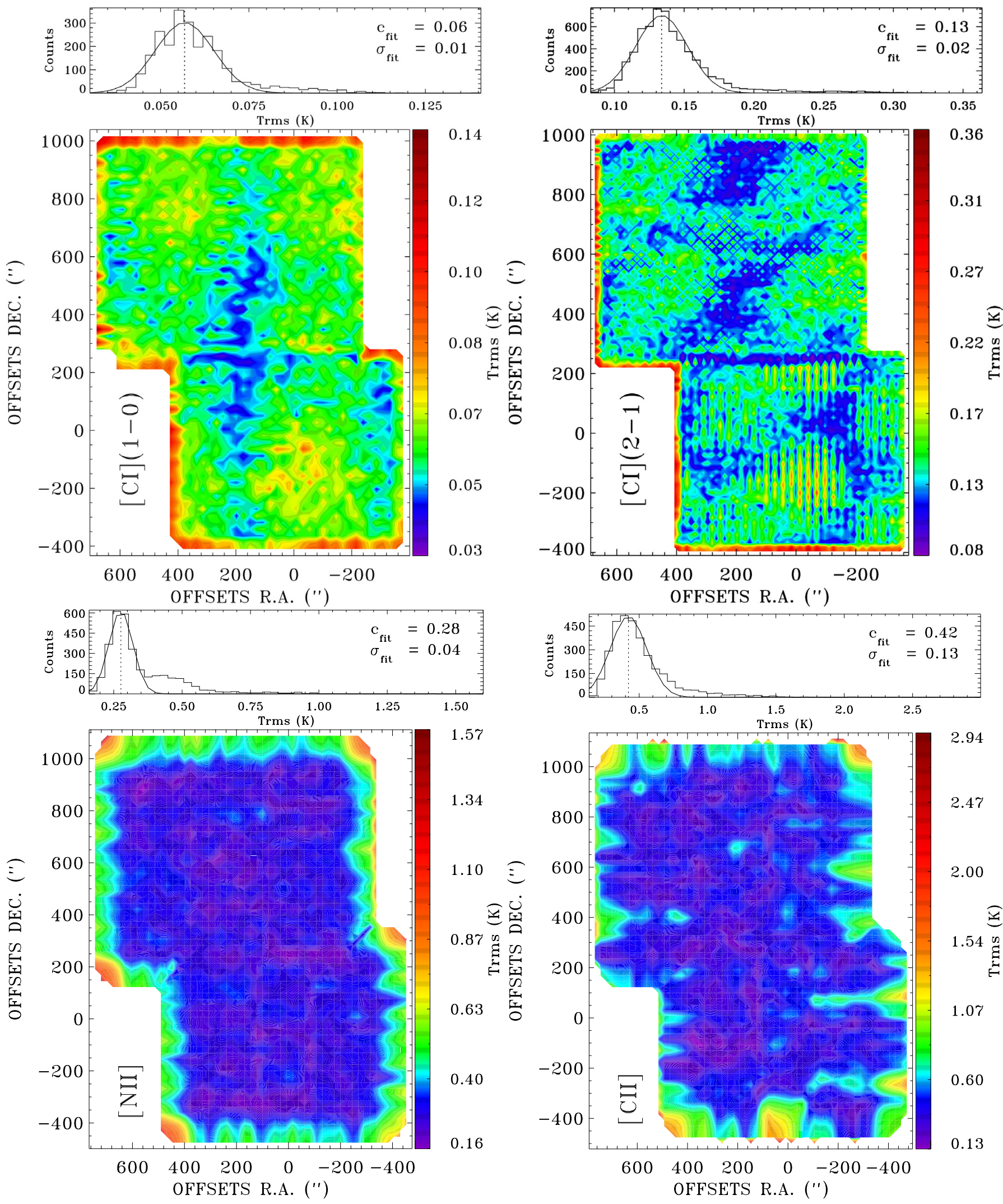

Fig. 7. $[\mathrm{CI}](1-0),[\mathrm{CI}](2-1),[\mathrm{NII}]$, and $[\mathrm{CII}] T_{A, \mathrm{rms}}^{*}$ noise distributions. For each panel, the spatial noise distribution as well as the histogram of the noise distribution are shown. The noise distribution spatial maps are centred at the $\alpha(\mathrm{J} 2000)=17^{\mathrm{h}} 45^{\prime} 39.9^{\prime \prime}, \delta(\mathrm{J} 2000)=-29^{\circ} 00^{\prime} 28.1^{\prime \prime}$ position. From the spatial maps, the noise is higher at the edges of the maps, as expected given the small number of spectra at the borders with which to calculate the resampled spectrum. From the histograms, a Gaussian fit (solid curve) shows the typical noise of each map $C_{\text {fit }}$ (Gauss centre shown as a vertical dashed line) and the standard deviation of the distributions $\sigma_{\text {fit }}$. 
measured essentially the same antenna temperatures within calibration errors, as expected. The typical pointing error of the $4 \mathrm{~m}$ NANTEN2/SMART telescope was within $\sim 10^{\prime \prime}$ and the absolute calibration error for the 460-490 GHz range, including uncertainties in beam efficiencies (Simon et al. 2007) and in the atmospheric calibration (see Sect. 2.2.2), is estimated to be around 20\% (Pineda et al. 2008, 2012).

\subsubsection{Observing strategy}

The observations of the $\mathrm{CO}(4-3)$ line were carried out in the Galactic coordinates reference frame using the OTF observing mode. The basic sampling unit of the SMART receiver, called the footprint, is a $340^{\prime \prime} \times 170^{\prime \prime}$ map that is Nyquist sampled at $810 \mathrm{GHz}$ and oversampled at $460 \mathrm{GHz}$. This spacing increases the signal-to-noise ratio of the lower frequency line after spatial resampling to the angular resolution consistent with the beam size. After each OTF line an OFF position measurement was performed, and a calibration load measurement was done every two OFF measurements.

The identification of a clean reference position for the observations is an enormous problem for large-scale surveys of the Galactic Centre. As previously mentioned, all HerschelHIFI observed lines suffered from emission contamination in the reference position that could be recovered largely because the receiver has only one pixel and no atmosphere had to be taken into account for the data calibration process. In the case of the NANTEN2/SMART observations, the picture is much more complex since during the OFF measurement each of the eight pixels at $460 \mathrm{GHz}$ points through the atmosphere at a different position on the sky, typically separated by $85^{\prime \prime}$. This means that not only a single clean position has to be found to be used as a reference but rather an area containing the observed positions in the reference measurement. Therefore, and based on the $\mathrm{CO}(2-1)$ observations of Sawada et al. (2001), the $l=+0^{\circ} .497$, $b=+0.980$ position was chosen. This position is located $\sim 1^{\circ}$ away from the Galactic Centre. In order to check whether there is still emission contamination, despite the great distance to the reference position, we observed the reference position against a more distant reference position that had been previously checked for emission contamination and found to be clean. The observations showed no emission within the footprint containing all eight pixels, at least down to a $3 \times T_{A, \text { rms }}^{*}$ significance level. Unfortunately, selecting a position far away from the astronomical source also has some drawbacks, especially in terms of the receiver stability and airmass between on-source and reference position measurements. This causes baseline distortions to appear in some of the calibrated spectra.

\subsubsection{SMART data reduction process}

In order to calibrate the observations, the atmospheric transmission as a function of frequency $t_{\mathrm{a}}(\tau(v))$ for each measured spectrum is needed. In order to determine $t_{\mathrm{a}}(\tau(v))$ in each side band, additional information of the atmosphere's behaviour is obtained from the atmospheric model (ATM) developed by Pardo et al. (2001), adapted for the telescope's altitude at $4865 \mathrm{~m}$ above sea level. From the model and the observations, the amount of precipitable water vapour $\left(m_{\mathrm{H}_{2} \mathrm{O}}\right)$ was fitted, the atmospheric opacity $\tau_{v} \propto m_{\mathrm{H}_{2} \mathrm{O}}$ was obtained, and the $t_{\mathrm{a}}(\tau(v))$ value was derived (Guan et al. 2012). After all observations were calibrated, the data were further reduced with the CLASS package where the spectra were brought to the forward beam antenna temperature

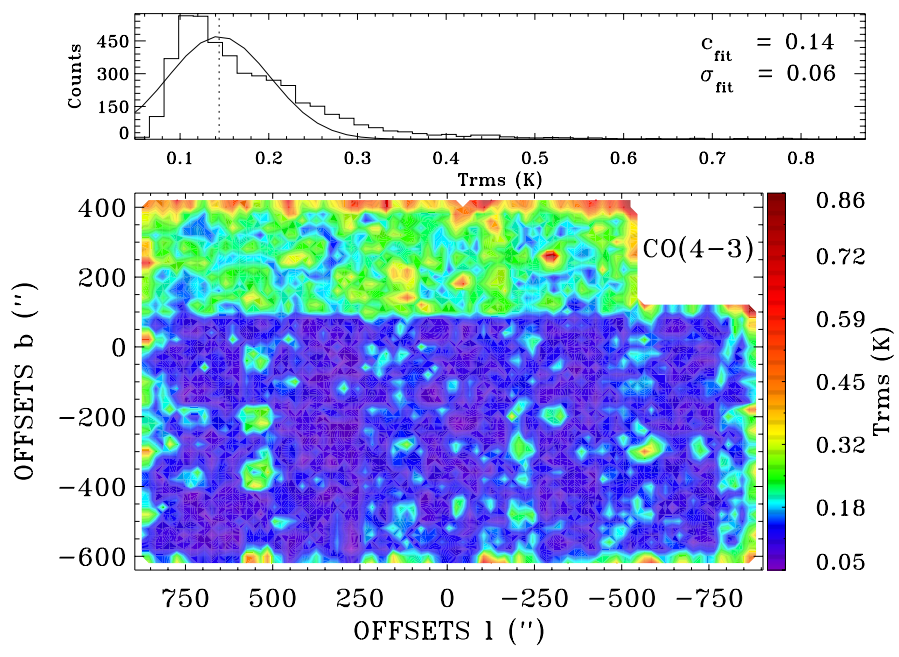

Fig. 8. $\mathrm{CO}(4-3) T_{A, \mathrm{rms}}^{*}$ noise distributions. The spatial noise distribution and the histogram of the noise distribution are shown. The noise distribution spatial map is centred at $l=0^{\circ}, b=0^{\circ}$. From the spatial map, the noise is higher at the edges of the map, as expected given the small number of spectra at the borders with which to calculate the resampled spectrum. From the histogram, a Gaussian fit (solid curve) shows the typical noise of the map $C_{\text {fit }}$ (Gauss centre shown as a vertical dashed line) and the standard deviation of the distribution $\sigma_{\text {fit }}$.

scale $T_{A}^{*}$ and low order (0 to 3 ) polynomials were fitted and subtracted from them (see Sect. 2.3.2 for an explanation on the selected antenna temperature scale). We only kept spectra with rms noise $T_{A, \mathrm{rms}}^{*}<2 \mathrm{~K}$, signal-sideband opacity $\tau_{\mathrm{s}}<0.7$ (which implies an attenuation of the sky signal by the atmosphere of about $50 \%$ ), and system temperature $T_{\text {sys }}<800 \mathrm{~K}$. Any spectrum with at least one of the three parameters above the corresponding thresholds was discarded. The final data cube was created with a spatial resolution $\left(40^{\prime \prime}\right)$ slightly lower than the beam size $\left(37.4^{\prime \prime}\right)$ at this frequency. The spatial noise distribution of the NANTEN2/SMART data is shown in Fig. 8.

\subsection{Antenna temperature scale and Herschel-HIFI- NANTEN2/SMART cross-calibration}

\subsubsection{Temperature scale}

In order to choose a common absolute antenna temperature scale for combining the NANTEN2/SMART and Herschel-HIFI observations, we examine the emission distribution of the Sgr A region and some aspects of the antenna patterns of both telescopes. Table 1 contains all the relevant parameters used to define the absolute temperature scales. The spatial extension of the submillimetre emission in the Sgr A region, as can be seen in García (2015), is very large compared to the beam sizes and side lobes in all lines included in the present work. For the Herschel satellite, Müller \& Jellema (2014) improved the analysis of the Herschel beam pattern done by Roelfsema et al. (2012) using all Mars observations performed during the Herschel operations together with an optical model of the telescope, which includes obscuration, truncation, and measured wave-front errors. They derived more accurate estimates for the beam sizes $\left(\Theta_{\mathrm{FWHM}}\right)$ and main beam efficiencies $\left(B_{\text {eff }}\right)$ for each measured polarization, while in Table 1 the quoted main beam efficiencies are the averaged values of the two polarizations. We kept the beam sizes estimated in Roelfsema et al. (2012), since the percentage differences between both works range only from $2 \%$ to $0 \%$ with increasing frequency. From the measured beam patterns, power 
distributions, and encircled energy fraction (EEF) in Müller \& Jellema (2014) there is a substantial fraction of the power in the side lobes that varies with frequency. This is shown by the slow convergence of the EEF at large radii $\left(>20 \times \Theta_{\text {FWHM }}\right)$. In a recent publication, Ossenkopf et al. (2013) used the antenna temperature scale for [CII] observations arguing for a large contribution from the side lobes to their extended measured emission. For the NANTEN2/SMART telescope, the error beam at $461.1 \mathrm{GHz}$ is expected to be within 7 to 10 times the size of the main lobe (Simon et al. 2007, Internal NANTEN2 Report), i.e. at radii less than $3^{\prime}$. The error beam is expected to contain around $20 \%$ of the detected power (U. Graf, priv. comm.). Given the extended emission of the source and the location of the error beams for both telescopes, we consider that the most representative temperature scale of the true convolved antenna temperatures within the Sgr A region is the Forward Beam Antenna Temperature $T_{A}^{*}$, where the corresponding forward efficiency $F_{\text {eff }}=\iint_{2 \pi} P(\Omega) \mathrm{d} \Omega / \iint_{4 \pi} P(\Omega) \mathrm{d} \Omega$ (Kramer 1997), with $P(\Omega)$ the antenna pattern of the telescope, has been applied. We apply this temperature scale to all data sets used in the present work. Given the contribution of the error beam pickup, the $T_{A}^{*}$ scale represents a lower limit to the true convolved antenna temperature. When the emission is more confined inside the respective error beam, the Main Beam Antenna Temperature scale, with the corresponding main beam (MB) efficiency $B_{\text {eff }}=\iint_{\text {MB }} P(\Omega) \mathrm{d} \Omega / \iint_{4 \pi} P(\Omega) \mathrm{d} \Omega$ (Kramer 1997), would imply a negligible contribution of the error beam pickup to the measured antenna temperatures, increasing the line strength by $\frac{F_{\text {eff }}}{B_{\text {eff }}}$.

\subsubsection{Cross-calibration}

The $[\mathrm{CI}](1-0)$ line was used to cross-check the antenna temperatures measured with both telescopes. A small area around the peak intensity, shown by the Herschel-HIFI observations, was selected and re-observed with the NANTEN2/SMART telescope. The spatial resolution of the map is 46" (smoothed to the Herschel-HIFI resolution) of a spacing of $23^{\prime \prime} \times 23^{\prime \prime}$. The spectral resolution of the data is $1 \mathrm{~km} \mathrm{~s}^{-1}$ and the rms noise temperature ranges from $0.044 \mathrm{~K}$ up to $0.071 \mathrm{~K}$. The number of spectra used for the cross-calibration around the emission peak is 20 . In order to compare the antenna temperatures measured with the two telescopes at the same positions on the sky, we explore the behaviour of the integrated intensity ratio with integration velocity interval. To minimize the influence of the noise on our results, we select only the emission that is above a $10 \sigma$ detection level, where $\sigma=0.071 \mathrm{~K}$ is the maximum rms noise in the observed spectra. This threshold defines a velocity range $\Delta V$ from $+23 \mathrm{~km} \mathrm{~s}^{-1}$ to $+83 \mathrm{~km} \mathrm{~s}^{-1}$ common for all spectra.

For each measured spectrum we divide $\Delta V$ into velocity intervals $\left(\Delta V_{i}\right)$ in order to calculate the total integrated intensity in each of them. The following values were used for $\Delta V_{i}: 1,2,3$, $4,5,6,10,15,20,30$, and $60 \mathrm{~km} \mathrm{~s}^{-1}$. The velocity range $\Delta V$ is divided into a given $\Delta V_{i}$ value, yielding $n=\frac{\Delta V}{\Delta V_{i}}$ sub-intervals. With these definitions, the integrated intensity ratio $R\left(\Delta V_{i}\right)_{j}$ in the $j$ sub-interval for the given $\Delta V_{i}$ value is calculated from the Herschel-HIFI $I\left(\Delta V_{i}\right)_{j}^{\mathrm{H}}$ and the NANTEN2/SMART $I\left(\Delta V_{i}\right)_{j}^{\mathrm{N}}$ integrated intensities as $R\left(\Delta V_{i}\right)_{j}=I\left(\Delta V_{i}\right)_{j}^{\mathrm{N}} / I\left(\Delta V_{i}\right)_{j}^{\mathrm{H}}$. Then, the average integrated intensity ratio $R\left(\Delta V_{i}\right)$ for the given $\Delta V_{i}$ interval is calculated as $R\left(\Delta V_{i}\right)=\frac{1}{n} \sum_{j=1}^{n} R\left(\Delta V_{i}\right)_{j}$ for each pair of spectra observed at position $k$.

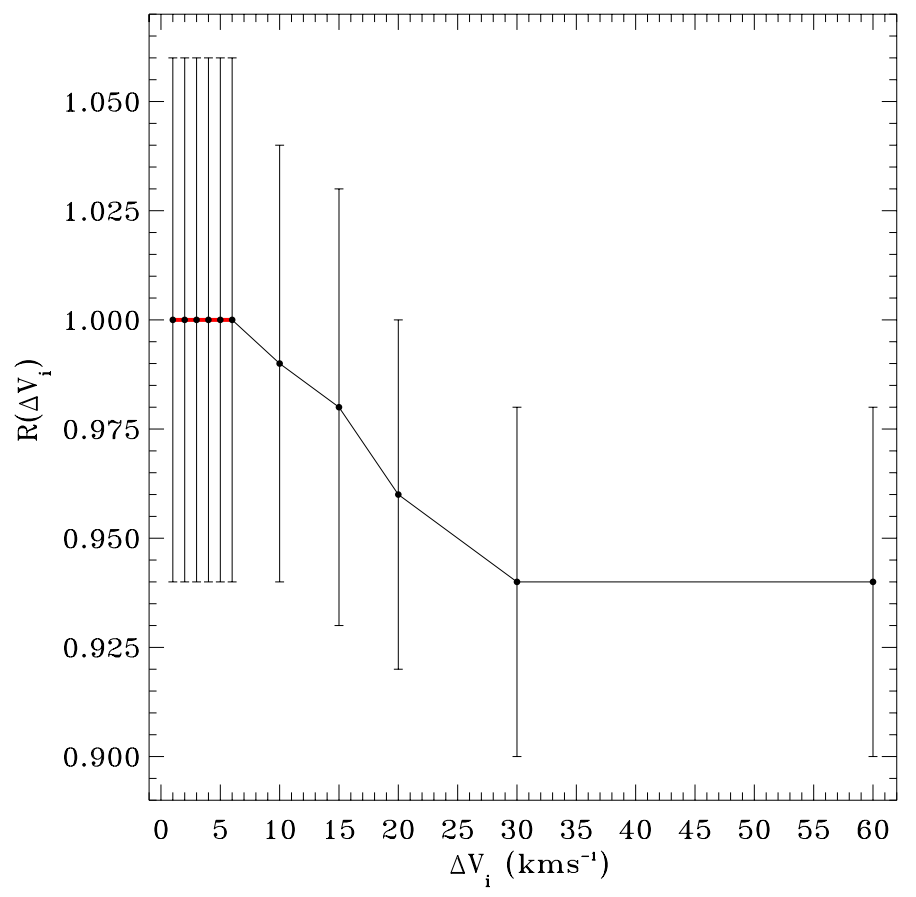

Fig. 9. Averaged integrated intensity ratio $R\left(\Delta V_{i}\right)$ of the [CI](1-0) emission measured with the Herschel-HIFI and NANTEN2/SMART telescopes as a function of velocity integration interval $\Delta V_{i}$ as explained in the text. The intensity ratios are on average $\sim 2 \%$ lower (within the error uncertainties) than unity over the whole range of $\Delta V_{i}$ values, showing that the measured NANTEN2/SMART antenna temperatures are systematically lower than those measured with the Herschel-HIFI telescope by the same amount. The red line delimits the $\Delta V_{i}$ range where the $R\left(\Delta V_{i}\right)$ values are closest to unity.

We combine the integrated intensity ratios $R\left(\Delta V_{i}\right)_{k}$ and $\Delta V_{i}$ information of all observed spectra. First, we calculate the averaged integrated intensity ratio for all spectra pairs at $\Delta V_{i}$ as $R\left(\Delta V_{i}\right)=\frac{1}{N} \sum_{k=1}^{N} R\left(\Delta V_{i}\right)_{k}$, with $N=20$. From this, we obtain one $R\left(\Delta V_{i}\right)$ value for a given $\Delta V_{i}$ interval. The results are shown in Fig. 9. The error bars correspond to the root-meansquare variation of each point and vary from $6 \%$ of $R\left(\Delta V_{i}\right)$ at $\Delta V_{i}=1 \mathrm{~km} \mathrm{~s}^{-1}$ to $4 \%$ at $\Delta V_{i}=60 \mathrm{~km} \mathrm{~s}^{-1}$.

From the figure, $R\left(\Delta V_{i}\right)$ is constant at 1.0 (red line) for $\Delta V_{i} \leq$ $6 \mathrm{~km} \mathrm{~s}^{-1}$. For larger $\Delta V_{i}$ values there is a small decrease to 0.94 , which could be attributed to differences in the error beam extent between the two telescopes, causing the measured antenna temperatures to be the result of the emission measured over slightly different spatial scales. The measured NANTEN2/SMART integrated antenna temperatures are consistently lower $(\sim 2 \%$ on average) than the measured quantities in the Herschel-HIFI observations. For comparison, assuming a calibration error of $20 \%$ in the absolute antenna temperature measured with both telescopes and an integrated intensity ratio of 0.98 , the corresponding error is $\sim 30 \%$, much larger than the $2 \%$ systematic underestimation of the Herschel-HIFI measured antenna temperatures by the NANTEN2/SMART measurements. Therefore, we are confident that both telescopes measure basically the same antenna temperatures on the $T_{A}^{*}$ scale; given the large spatial extension of the emission, we consider this a fair representation of the true convolved antenna temperature of the source. 


\subsubsection{ASTE $[\mathrm{CI}] 1-0 \mathrm{CMZ}$ survey}

Recently, Tanaka et al. (2011) carried out a 34" angular resolution $[\mathrm{CI}](1-0)$ Survey of the CMZ using the $10 \mathrm{~m}$ Atacama Sub-millimeter Telescope Experiment (ASTE) telescope. At a spatial resolution of $46^{\prime \prime}$ and spectral resolution of $2 \mathrm{~km} \mathrm{~s}^{-1}$, the $[\mathrm{CI}](1-0)$ peak intensity in their data set is $10.9 \pm 0.2 \mathrm{~K}$, at $(l, b)=(-0.02,-0.07)$, at LSR velocity $+53 \mathrm{~km} \mathrm{~s}^{-1}(\mathrm{~K}$. Tanaka, priv. comm.) in the main beam antenna temperature scale $\left(B_{\text {eff }}=0.50\right)$. From $800 \mathrm{GHz}$ measurements, the forward efficiency of the ASTE is considered to be $F_{\text {eff }} \sim 1.0$ and the error beam pickup was estimated to be $10 \%$ of the power peak detected by the antenna. The absolute calibration error is estimated to be around $8 \%$. In the $T_{A}^{*}$ antenna temperature scale, their peak intensity reduces to $5.45 \pm 0.10 \mathrm{~K}$.

The peak intensity of Herschel-HIFI [CI](1-0) observations with $2 \mathrm{~km} \mathrm{~s}^{-1}$ spectral resolution and $46^{\prime \prime}$ spatial resolution is $8.13 \pm 0.05 \mathrm{~K}$ in $T_{A}^{*}$ temperature scale, at position $(l, b)=$ $\left(-0^{\circ} .017,-0^{\circ} .071\right)$ in Galactic coordinates and at LSR velocity $+53 \mathrm{~km} \mathrm{~s}^{-1}$. In the case of the [CI](1-0) NANTEN2/SMART observations used for the cross-calibration with the HerschelHIFI data, at the same position and LSR velocity, we measured $6.09 \pm 0.06 \mathrm{~K}$ in $T_{A}^{*}$ temperature scale. This value is consistent with the Herschel-HIFI value within error uncertainties when considering the absolute calibration error. When considering the absolute calibration errors in the Herschel and ASTE data sets, the measured intensity peaks in $T_{A}^{*}$ scale are not consistent within error uncertainties. Nonetheless, we noticed that a lower forward efficiency in the case of the ASTE telescope would bring their measured intensity peak closer to the Herschel-HIFI value, and since $F_{\text {eff }}$ was derived only from $800 \mathrm{GHz}$ measurements, this could indeed be the case for the $492 \mathrm{GHz}$ observations.

\subsection{Observations summary}

A summary of the main parameters describing the scientific version data sets of all observed lines is presented in Table 1. The central position of all Herschel-HIFI observed maps is $\alpha(\mathrm{J} 2000)=17^{\mathrm{h}} 45^{\prime} 39.9^{\prime \prime}, \delta(J 2000)=-29^{\circ} 00^{\prime} 28.1^{\prime \prime}$ in equatorial coordinates, while the NANTEN2/SMART observations are centred at $l=0.000, b=0.000$ in Galactic coordinates for historical reasons (observations started as a larger CMZ survey). The columns in Table 1 counted from left to right contain telescope name; observed line name; transition frequency $\left(v_{\mathrm{obs}}\right)$; telescope beam size at the observed frequency $\left(\Theta_{\mathrm{FWHM}}\right)$ taken from Roelfsema et al. (2012) in the case of HerschelHIFI observations; final spatial resolution of the data set $(\Theta)$; forward efficiency of the telescope $\left(F_{\text {eff }}\right)$; main beam efficiency of the telescope $\left(B_{\text {eff }}\right)$ from Müller \& Jellema (2014) in the case of Herschel-HIFI observations; typical noise in the data set $\left(T_{A, \text { rms }}^{*}\right)$; original LSR velocity resolution $\left(\Delta V_{\text {res }}\right)$; resampled spectral resolution $\left(\Delta V_{f}\right)$; LSR velocity coverage of the observations $\left(\Delta V_{\text {range }}\right)$; and energy of the upper level above the ground state for the observed line $\left(E_{\mathrm{u}}\right)$. In the next section, the morphology of the emission in all lines as a function of LSR velocity is described and discussed.

\section{Emission morphology}

In the following, we discuss the spatial distribution of the emission in all lines, and across the $\pm 200 \mathrm{~km} \mathrm{~s}^{-1}$ LSR velocity range in our data. The data are presented in channel maps of integrated intensity in units of $\mathrm{K} \mathrm{km} \mathrm{s}^{-1}\left(I=\int T_{A}^{*}(v) \mathrm{d} v\right)$. For each map, the central LSR velocity is given. The spatial resolution in each data set is shown as a filled black circle in each map. For all observations, the right ascension (RA) and declination (Dec) offsets, and Galactic longitude $(l)$ and Galactic latitude $(b)$ offsets are with respect to the central position mentioned in Sect. 2.4. The channel maps can be found in García (2015). Given the large spatial and angular extent of our data sets, extra channel or integrated intensity maps are displayed when necessary for a better overview of the discussion of particular astronomical sources. In those figures, the NANTEN/SMART data have been rotated to the equatorial (J2000) coordinate system for better comparison with the Herschel-HIFI observations.

As described in Sect. 1, the Sgr A Complex contains an enormous variety of astronomical sources. In order to give a better overview of the region and the sources within it, we have compiled a number of relevant sources from the literature for the discussion on the morphology of the region in our sub-mm/FIR observations (Güsten et al. 1981; Serabyn \& Guesten 1987; Yusef-Zadeh \& Morris 1987a,b; Zhao et al. 1993; Cotera et al. 1996; Timmermann et al. 1996; Lang et al. 1997, 1999a,b, 2001, 2002, 2010; Porquet et al. 2003; Oka et al. 2008; Caswell et al. 2010, 2011; Requena-Torres et al. 2012). Figure 10 shows the $20 \mathrm{~cm}$ continuum emission observed by Yusef-Zadeh \& Morris (1987a) delineating large-scale structures and point sources. The figure includes the position of the massive black hole Sgr A* (very close to which the massive Nuclear cluster is found), the AI and M H II regions close to the GC; the Sgr A-East and Sgr AWest regions; the Radio Arc and other non-thermal structures such as the Northern and Southern Threads; the Thermal Arched Filaments E1, E2, W1, W2; the "banana" G0.10+0.02 and G0.07+0.04 structures; the positions of the massive Quintuplet and Arches clusters, and of the Pistol star (G0.15-0.05); the extended Sickle H II region, several H II regions (H+number) forming the H Region; water and methanol masers; and the Xray source $1 \mathrm{E} 1743.1-2843 \mathrm{~B}$. The dotted-line in the map shows the area covered by the Herschel-HIFI observations while the solid straight line indicates the Galactic plane at $b=0^{\circ}$. In order to have an overview of the molecular material in the Sgr A Complex, Fig. 11 shows the integrated intensity map (between $-75 \mathrm{~km} \mathrm{~s}^{-1}$ and $+110 \mathrm{~km} \mathrm{~s}^{-1}$ ) of $\mathrm{N}_{2} \mathrm{H}^{+}(1-0)$ (diazenylium) of the Mopra observations by Jones et al. (2012), tracing cold and dense gas. In this figure, the position of all the major molecular clouds (including the "Brick" M+0.25+0.01), the HVCC (CO+0.02-0.02), and the extent of the CND (black square), are shown. The diversity of large structures seen in Figs. 10 and 11 shows the need of multiwavelength comparison in order to understand the large-scale spatial and spectral distribution of the warm gas component traced in our data.

\subsection{Average spectrum of the observed sub-mm lines}

The average spectrum of the observed submillimetre lines is shown in Fig. 12. The spectra were obtained by bringing all data sets to a common $46^{\prime \prime}$ angular resolution and averaging all spectra within the area covered by the Herschel-HIFI observations. If one considers the average spectrum as a single-point observation, it would be roughly equivalent to a spectrum taken with a spatial resolution of $\approx 50 \mathrm{pc}$. The bulk of the carbon monoxide and atomic carbon emission is concentrated in the major MCs towards positive LSR velocities, while the emission of the ionized material is more symmetric with respect to $0 \mathrm{~km} \mathrm{~s}^{-1}$. Given the low rms noise of the Herschel-HIFI and NANTEN2/SMART observations, even weak emission at very high LSR velocities $\left(V_{\mathrm{lsr}}>+150 \mathrm{~km} \mathrm{~s}^{-1}\right)$ in the $[\mathrm{CI}](1-0)$ and $\mathrm{CO}(4-3)$ lines is detected in the average spectrum. Absorption features along the 
P. García et al.: Warm ISM in the Sagittarius A Complex. I.

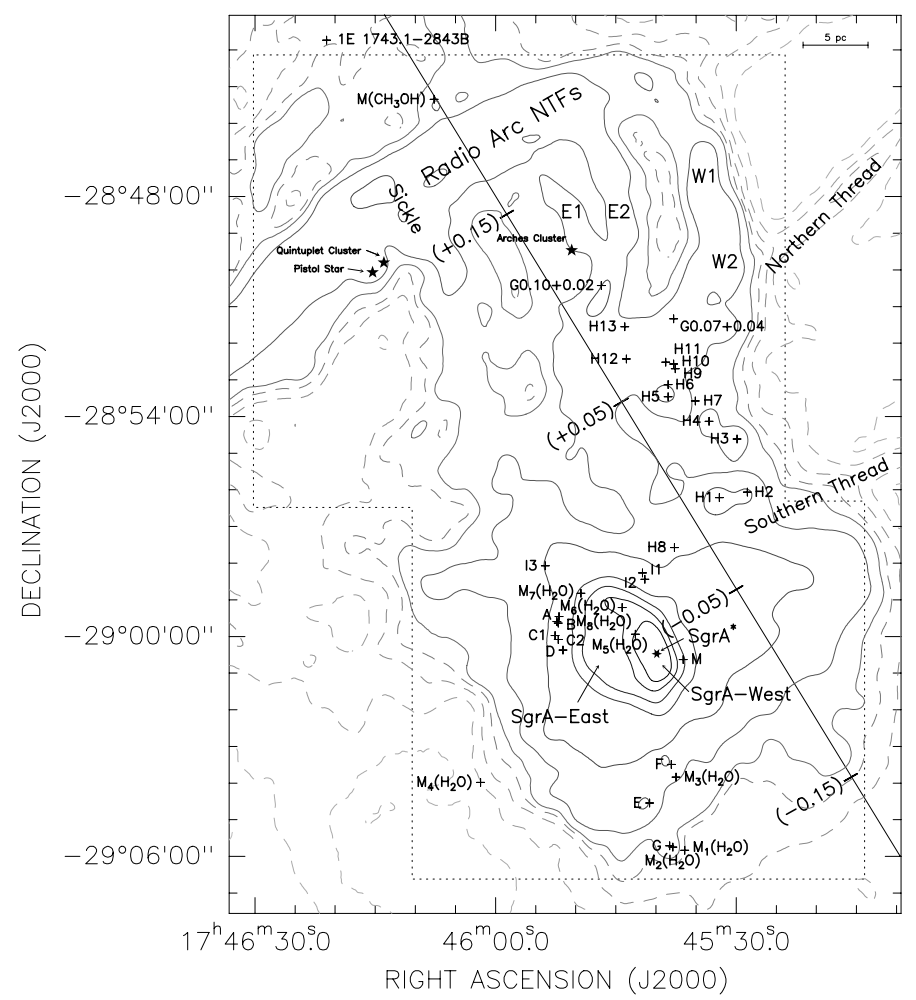

Fig. 10. $20 \mathrm{~cm}$ continuum observations at $30^{\prime \prime}$ spatial resolution from Yusef-Zadeh \& Morris (1987a). Overplotted on the figure are several discrete sources found in the literature (see Sect. 3). Following Lang et al. (2010), contours levels are at 10, 15, 20, 25, 30, 35, 50, 100, 300, 500, 700, and 900 times $8.5 \mathrm{mJy}_{\text {beam }}{ }^{-1}$. The solid straight line shows the position of the Galactic plane at $b=0^{\circ}$, with marks denoting a few Galactic longitude values for orientation. The dotted line shows the area covered by the Herschel-HIFI observations.

1.o.s. associated with the loci of spiral arms are observed in the $\mathrm{CO}(4-3)$ average spectrum: at $-5 \mathrm{~km} \mathrm{~s}^{-1}$ the most prominent absorption feature associated with the local arm is seen, while other weaker absorption features at $-30 \mathrm{~km} \mathrm{~s}^{-1}$ and $-55 \mathrm{~km} \mathrm{~s}^{-1}$ are associated with the $3 \mathrm{kpc}$ and $4.5 \mathrm{kpc}$ spiral arms (Oka et al. 1998; Jones et al. 2012; Dame et al. 2001; Dame \& Thaddeus 2008; García et al. 2014). The average [CII] spectrum also shows some correspondence with the absorption LSR velocity of the spiral arms along the l.o.s., while no clear absorption signatures are seen in the carbon average spectra. It is important to notice that the absorption due to foreground spiral arms is expected to be narrow with $\Delta V \leq 5 \mathrm{~km} \mathrm{~s}^{-1}$ for the $\mathrm{CO}(1-0)$ transition (Oka et al. 1998). Therefore, broader absorption features in the warm gas could (at least partially) originate from in situ absorption. For instance, the absorption feature at $-5 \mathrm{~km} \mathrm{~s}^{-1}$ seen in the average $\mathrm{CO}(4-3)$ spectrum has a line width $\Delta V \approx 10 \mathrm{~km} \mathrm{~s}^{-1}$.

\subsection{Molecular Cloud Complexes between $-80 \mathrm{~km} \mathrm{~s}^{-1}$ up to $+90 \mathrm{~km} \mathrm{~s}^{-1}$}

Most of the molecular clouds within the Sgr A Complex are located between $-80 \mathrm{~km} \mathrm{~s}^{-1}$ and $+90 \mathrm{~km} \mathrm{~s}^{-1}$. From negative to positive LSR velocities, the first feature is $\mathrm{M}+0.04+0.03$ ( $-30 \mathrm{~km} \mathrm{~s}^{-1}$ cloud), which contains the gas associated with the Arched Filaments (Serabyn \& Guesten 1987; Zhao et al. 1993) ranging from $\sim-70 \mathrm{~km} \mathrm{~s}^{-1}$ up to $0 \mathrm{~km} \mathrm{~s}^{-1}$ and covering the upper half of the Herschel-HIFI maps. Figure 13 (upper panels) shows $5 \mathrm{~km} \mathrm{~s}^{-1}$ integrated intensity maps of the [CII] (colour

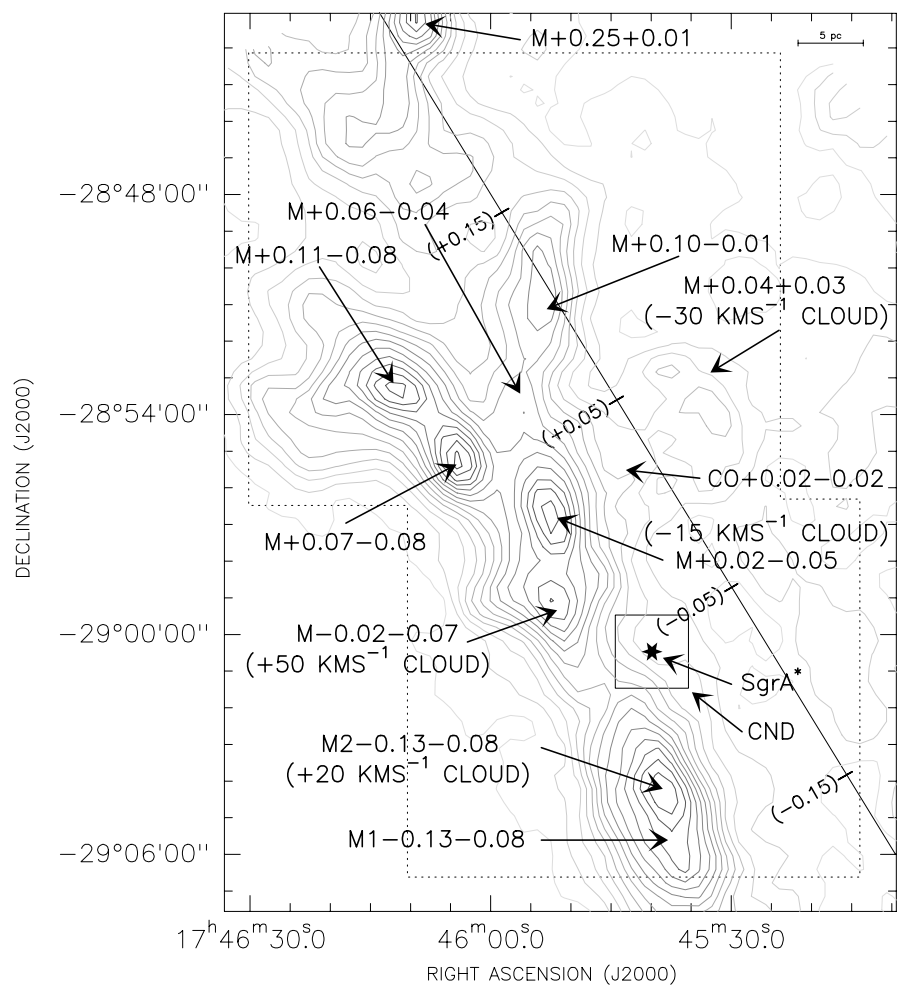

Fig. 11. $\mathrm{N}_{2} \mathrm{H}^{+}(1-0)$ (diazenylium) integrated intensity map between -75 and $+110 \mathrm{~km} \mathrm{~s}^{-1}$ of the Mopra observations by Jones et al. (2012). The spatial resolution of the map is $44^{\prime \prime}$ with $3.6 \mathrm{~km} \mathrm{~s}^{-1}$ spectral resolution. The first contour is located at $\sim 1 \mathrm{~K} \mathrm{~km} \mathrm{~s}^{-1}$, equivalent to $5 \times \sigma$ with $\sigma$ the typical noise of the map. The contour increment is $5 \mathrm{~K} \mathrm{~km} \mathrm{~s}^{-1}$. The solid straight line shows the position of the Galactic plane at $b=0^{\circ}$; the marks denote a few Galactic longitude values for orientation. The area is covered by the Herschel-HIFI observations.

scale) and [NII] (contours) emission with the corresponding central LSR velocity of the maps shown in the bottom of each panel. The red squares depict the positions of two peaks in CS(2-1) emission detected by Serabyn \& Guesten (1987), tracing high density material, while the red star shows the position of the massive Arches Cluster. The emission in both lines is very widespread and closely follows the $20 \mathrm{~cm}$ continuum emission shown in Fig. 10, moving from the H Region, with a local intensity peak in both lines at the position of the H1-H2 sources, to the Arched Filaments, going through G0.07+0.04, and moving from the W1/W2 filaments to the E1/E2 filaments and the "banana" as the LSR velocity approaches $0 \mathrm{~km} \mathrm{~s}^{-1}$. From the maps, a strong spatial correlation between the [CII] and [NII] lines is observed, indicating that there is a significant contribution to the observed $[\mathrm{CII}]$ emission from the $\mathrm{H}$ II regions and not only from PDRs (Abel et al. 2005). The case of G0.07+0.04 is particularly interesting since it is thought that at this position the gas is interacting with the Northern Thread (Lang et al. 1999b). In a preliminary analysis, the central LSR velocity of the H92 $\alpha$ average spectrum in this region shown by Lang et al. (2001) is shifted by around $\sim 30 \mathrm{~km} \mathrm{~s}^{-1}$ towards more negative velocities with respect to the bulk of carbon and carbon monoxide emission along the same 1.o.s. Such a large velocity shift is not seen at other positions of the Arched Filaments, and might indicate that the ionized material is drifting away from G0.07+0.04 as a result of the interaction with the local magnet field.

The peak $[\mathrm{CII}]$ intensity $(\sim 9.2 \pm 0.4 \mathrm{~K})$ occurs within the Arched Filaments at $\Delta \alpha(\mathrm{J} 2000)=82.2^{\prime \prime}, \Delta \delta(\mathrm{J} 2000)=+490.4^{\prime \prime}$, 


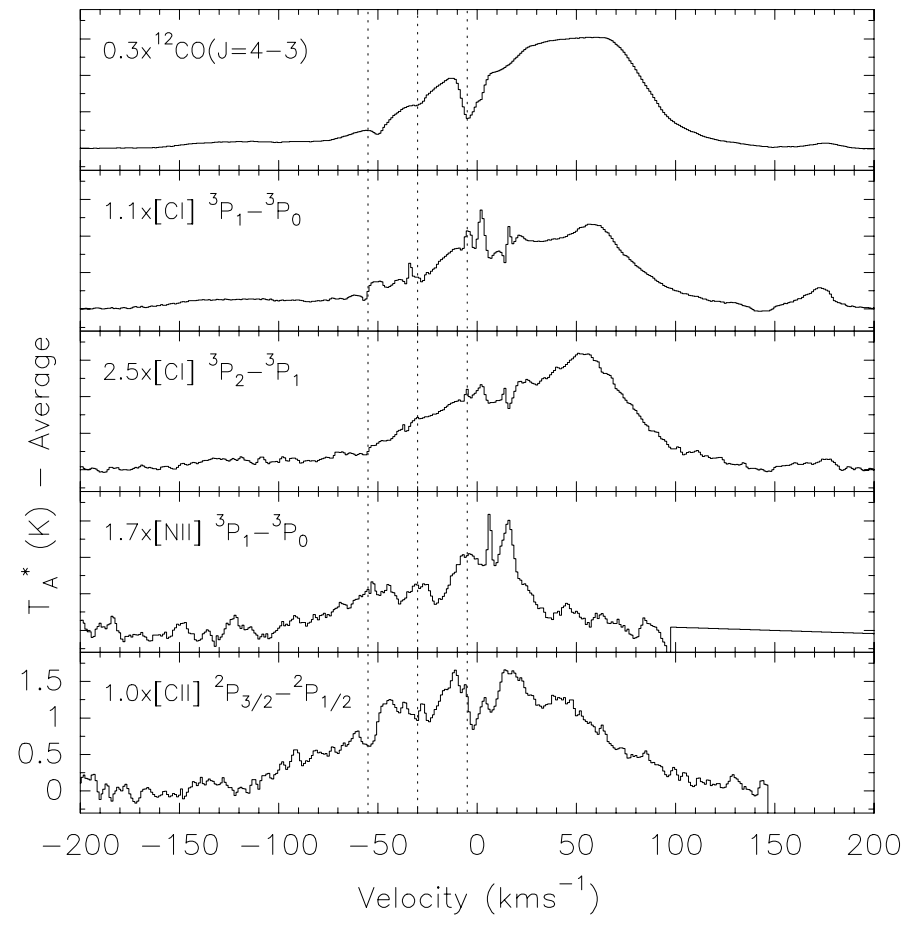

Fig. 12. Average spectra of the sub-mm/FIR lines observed in the present work. Each average spectrum is made out of $46^{\prime \prime}$ spatial resolution data cubes and within the area covered by the Herschel-HIFI observations. Vertical dotted lines show the loci of the $3 \mathrm{kpc}, 4.5 \mathrm{kpc}$, and local arm spiral features at $\sim-55 \mathrm{~km} \mathrm{~s}^{-1}, \sim-30 \mathrm{~km} \mathrm{~s}^{-1}$, and $\sim-5 \mathrm{~km} \mathrm{~s}^{-1}$, respectively.

and $V_{\mathrm{LSR}}=-45 \mathrm{~km} \mathrm{~s}^{-1}$, while the peak intensity of the [NII] line $(\sim 2.7 \pm 0.4 \mathrm{~K})$ occurs at $\Delta \alpha(\mathrm{J} 2000)=-107.2^{\prime \prime}, \Delta \delta(\mathrm{J} 2000)=$ $+720.3^{\prime \prime}$, and $V_{\mathrm{LSR}}=-26 \mathrm{~km} \mathrm{~s}^{-1}$. Figure 13 (bottom panels) shows the emission distribution of the $\mathrm{CO}(4-3)$ and $[\mathrm{CI}](1-0)$ The behaviour of the $[\mathrm{CI}](2-1)$ line is very similar to the $[\mathrm{CI}](1-0)$ emission (as expected), so we focus the discussion on the lower frequency line. At large negative LSR velocities, there is a gas streamer connecting the $\mathrm{H}$ region with the lower part of the Arched Filaments, where one of the CS(2-1) peaks is located (see Sect. 3.3). The bulk of the emission detected from the $\mathrm{CO}(4-3)$ and $[\mathrm{CI}](1-0)$ lines is displaced with respect to the position of the filaments and with respect to the $\operatorname{CS}(2-1)$ emission peaks detected by Serabyn \& Guesten (1987), and it is weak in comparison to the rest of the emission in the data cubes. The position of the most intense emission in the $\mathrm{CO}(4-3)$ line is at the centre of the maps, as seen for example at $-18 \mathrm{~km} \mathrm{~s}^{-1}$, between $\mathrm{M}+0.04+0.03\left(-30 \mathrm{~km} \mathrm{~s}^{-1}\right.$ cloud) and $\mathrm{M}+0.02-0.05$ $\left(-15 \mathrm{~km} \mathrm{~s}^{-1}\right.$ cloud), while local maxima of the carbon emission coincide with the position of these two clouds. In the top panels of Fig. 14, at $-11 \mathrm{~km} \mathrm{~s}^{-1}$, a lane of emission (both in carbon and carbon monoxide) is seen going from the position of $\mathrm{M}+0.02-0.05$ through the Galactic Centre and connecting with another cloud south of the CND, which is probably part of the $+20 \mathrm{~km} \mathrm{~s}^{-1}$ cloud. Serabyn \& Guesten (1987) suggested that the lack of ionized material in the region around $\mathrm{M}+0.02-0.05$, the so-called molecular bridge, could be the result of the tidal disruption of material in infall towards the CND. This seems to be supported by the morphology of the warm gas in our observations (see Sect. 3.3).

From $0 \mathrm{~km} \mathrm{~s}^{-1}$ to $+90 \mathrm{~km} \mathrm{~s}^{-1}$, there is a large number of molecular clouds outlined in different temperature and density tracers at lower frequencies. We refer to them following the nomenclature used by Güsten et al. (1981) who identified their $\mathrm{NH}_{3}(1,1)$ peak positions, as we did in Fig. 11. Given the molecular nature of the MCs, these are brighter in the $\mathrm{CO}(4-3)$ emission than in any other line in our data. From $0 \mathrm{~km} \mathrm{~s}^{-1}$ to $+20 \mathrm{~km} \mathrm{~s}^{-1}$, the brightest feature is the M2-0.13-0.08 $\left(+20 \mathrm{~km} \mathrm{~s}^{-1}\right.$ cloud) seen at $\Delta l<0^{\prime \prime}$. The emission lane that crosses the GC from the molecular bridge to connect with the $+20 \mathrm{~km} \mathrm{~s}^{-1}$ cloud is still clearly visible at $+8 \mathrm{~km} \mathrm{~s}^{-1}$, as seen in the bottom panels of Fig. 14. These streamers are traced almost uninterruptedly by the $[\mathrm{CI}](1-0)$ and the $[\mathrm{CI}](2-1)$ emission, since no absorption features are present in these lines within the $-11 \mathrm{~km} \mathrm{~s}^{-1}$ to $+8 \mathrm{~km} \mathrm{~s}^{-1}$ LSR velocity range. From the morphological point of view, this suggests that the molecular bridge and the $+20 \mathrm{~km} \mathrm{~s}^{-1}$ cloud are part of the same structure that is being tidally disrupted in the gravitational field of the Nuclear Cluster, which dominates the gravitational potential for radii $2-30 \mathrm{pc}$ (Longmore et al. 2013). In this case, the emission gap between the bridge and the $+20 \mathrm{~km} \mathrm{~s}^{-1}$ cloud in the $\mathrm{CO}(4-3)$ emission is, at least partially, due the strong absorption of the local arm along the l.o.s.

For $\Delta l>0^{\prime \prime}$, two $\mathrm{CO}(4-3)$ emission features appear at $\Delta l=+400^{\prime \prime}, \Delta b=-400^{\prime \prime}$, and $\Delta l=+750^{\prime}, \Delta b=-150^{\prime \prime}$. The former surrounds the $\mathrm{M}+0.11-0.08$ cloud as seen in $\mathrm{N}_{2} \mathrm{H}^{+}(1-0)$ emission from the Mopra observations (Jones et al. 2012), and it is associated with the M+0.11-0.11 dense cloud observed in $\operatorname{CS}(1-0)$ and $\operatorname{CS}(2-1)$ by Tsuboi et al. (1997), while the latter is slightly shifted with respect to a local $\mathrm{N}_{2} \mathrm{H}^{+}(1-0)$ peak, not covered in the Güsten et al. (1981) observations, and coincides spatially with the edge of the Sickle H II region. Both features anti-correlate with the $20 \mathrm{~cm}$ continuum emission measured by Yusef-Zadeh \& Morris (1987a). This is illustrated by the $3 \mathrm{~km} \mathrm{~s}^{-1}$ wide $\mathrm{CO}(4-3)$ integrated intensity map shown in Fig. 15 at central LSR velocity $+29 \mathrm{~km} \mathrm{~s}^{-1}$. The contours in the figure show the Sickle region depicted in the continuum emission (left panel), the $\mathrm{M}+0.11-0.08$ cloud traced by the diazenylium line, and the position of the $\mathrm{M}+0.11-0.11$ cloud (right panel). Their $\mathrm{CO}(4-3)$ emission extends up to $\sim+40 \mathrm{~km} \mathrm{~s}^{-1}$ where these structures are still recognizable. The atomic carbon line observations follow the same trend as the carbon monoxide observations in this LSR velocity range. There are $[\mathrm{CII}]$ and $[\mathrm{NII}]$ detections towards the Sickle H II region, while no clear detection is seen towards the $\mathrm{M}+0.11-0.08$ cloud. In general, the [CII] emission is widespread, while the [NII] emission anti-correlates spatially at some spots with the lower frequency lines, as can be expected from gas adjacent to $\mathrm{H}$ II regions. An example of this is seen in the channel maps at LSR velocity $+18 \mathrm{~km} \mathrm{~s}^{-1}$, where a local maximum in the $[\mathrm{NII}]$ emission is located just next to the $\mathrm{CO}(4-3)$ emission of the $+20 \mathrm{~km} \mathrm{~s}^{-1}$ cloud at $\Delta \alpha(\mathrm{J} 2000) \sim-150^{\prime \prime}$, $\Delta \delta(\mathrm{J} 2000) \sim-250^{\prime \prime}$.

Most of the neutral atomic carbon emission and molecular emission is found within the LSR velocity range $+20 \mathrm{~km} \mathrm{~s}^{-1}$ up to $+90 \mathrm{~km} \mathrm{~s}^{-1}$. The peak intensities measured in our data sets are $T_{A}^{*}=22.11 \pm 0.09 \mathrm{~K}$ at $\Delta l=-64.9^{\prime \prime}, \Delta b=-259.3^{\prime \prime}$, and $V_{\mathrm{LSR}}=+63 \mathrm{~km} \mathrm{~s}^{-1}$ for the $\mathrm{CO}(4-3)$ line; $T_{A}^{*}=8.14 \pm 0.05 \mathrm{~K}$ at $\Delta \alpha(\mathrm{J} 2000)=+152.1^{\prime \prime}, \Delta \delta(\mathrm{J} 2000)=+73.0^{\prime \prime}$, and $V_{\mathrm{LSR}}=$ $+53 \mathrm{~km} \mathrm{~s}^{-1}$ for the $[\mathrm{CI}](1-0)$ line; and $T_{A}^{*}=6.98 \pm 0.13 \mathrm{~K}$ at $\Delta \alpha(\mathrm{J} 2000)=+155.2^{\prime \prime}, \Delta \delta(\mathrm{J} 2000)=+80.1^{\prime \prime}$, and $V_{\mathrm{LSR}}=$ $+55 \mathrm{~km} \mathrm{~s}^{-1}$ for the $[\mathrm{CI}](2-1)$ line, all three associated in space and LSR velocity with $\mathrm{M}-0.02-0.07\left(+50 \mathrm{~km} \mathrm{~s}^{-1}\right.$ cloud). For $\Delta l<+150^{\prime \prime}$, when going from $+20 \mathrm{~km} \mathrm{~s}^{-1}$ to $+50 \mathrm{~km} \mathrm{~s}^{-1}$, the $\mathrm{CO}(4-3)$ emission moves across Galactic longitude from the $+20 \mathrm{~km} \mathrm{~s}^{-1}$ cloud $\left(\Delta l=-350^{\prime \prime}, \Delta b=-250^{\prime \prime}\right)$ continuously to connect with the $+50 \mathrm{~km} \mathrm{~s}^{-1}$ cloud at around $\Delta l=-350^{\prime \prime}$, $\Delta b=-250^{\prime \prime}$. This behaviour is also seen in the atomic carbon 
P. García et al.: Warm ISM in the Sagittarius A Complex. I.
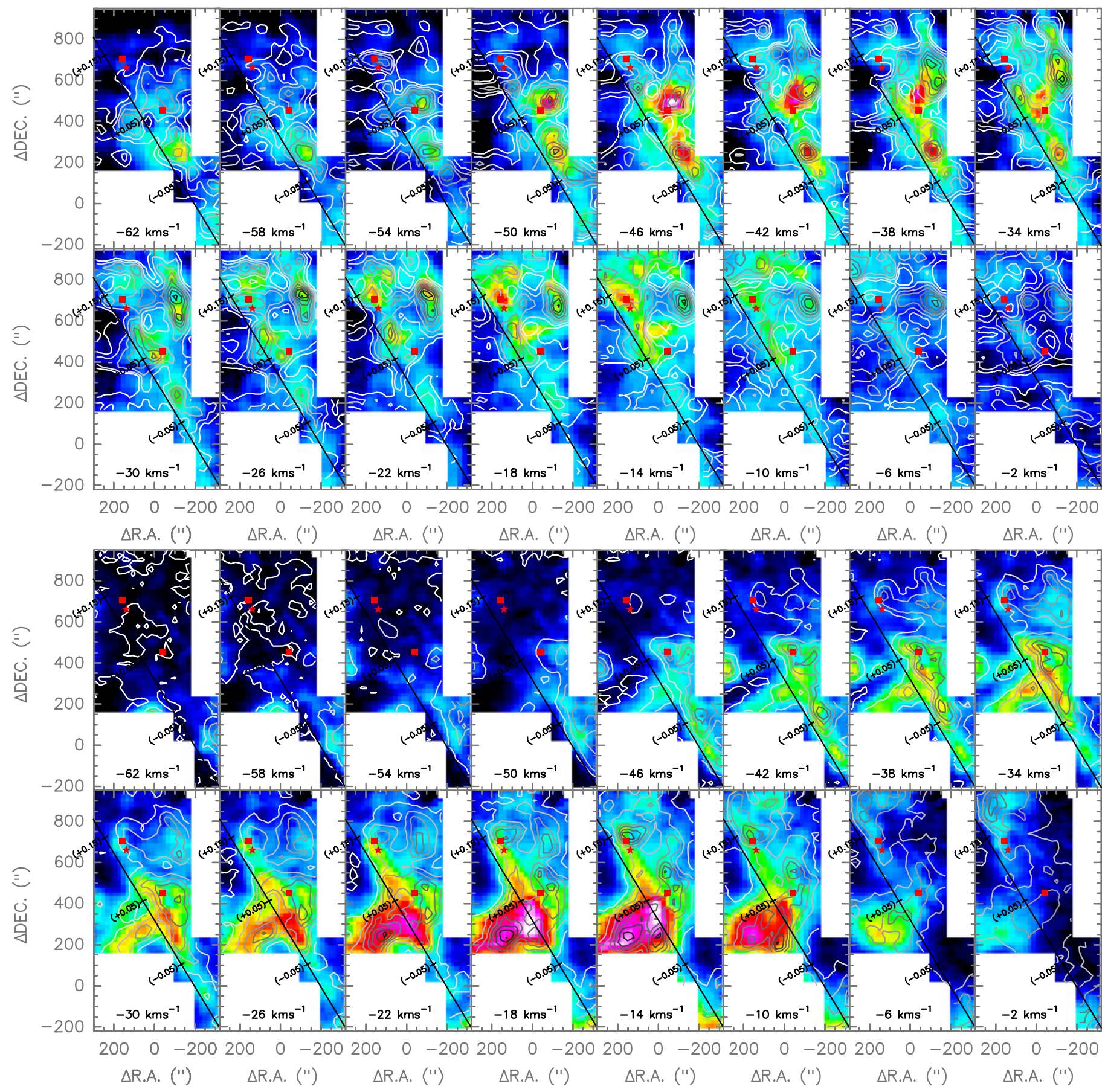

Fig. 13. Integrated intensity maps $\left(5 \mathrm{~km} \mathrm{~s}^{-1}\right.$ wide) of the [CII] (colour scale) and [NII] (contours) lines in the top panels and of the $\mathrm{CO}(4-3)$ (colour scale) and $[\mathrm{CI}](1-0)$ (contours) lines in the bottom panels, tracing the molecular and atomic material towards the Arched Filaments E1, E2, W1, and W2 and the H Region as shown in Fig. 12. The red star shows the location of the Arches Cluster, while red squares show the positions of two CS(2-1) peaks shown in the work by Serabyn \& Guesten (1987), tracing high density material. The solid straight line shows the position of the Galactic plane at $b=0^{\circ}$, with marks denoting a few Galactic longitude values for orientation. The central LSR velocity of the maps is shown in each panel.

lines from which, at LSR velocities between $+50 \mathrm{~km} \mathrm{~s}^{-1}$ and $+60 \mathrm{~km} \mathrm{~s}^{-1}$, the $+50 \mathrm{~km} \mathrm{~s}^{-1}$ cloud stands out as a crescentshaped feature with its concave side oriented to the non-thermal shell source Sgr A-East, which is thought to be the remnant of a supernova explosion (or explosions) with energy $\sim 4 \times 10^{52} \mathrm{erg}$ (Yusef-Zadeh \& Morris 1987a; Mezger et al. 1989; Coil \& Ho 2000). At $+67 \mathrm{~km} \mathrm{~s}^{-1}$, a similar crescent-shaped structure in $\mathrm{CO}(4-3)$ emission is seen. This is shown in Fig. 16, where the contours show the $20 \mathrm{~cm}$ continuum emission and the position of $\mathrm{Sgr} \mathrm{A}^{\star}$ is represented by the $\star$ symbol. This indicates that the emission detected from the $+50 \mathrm{~km} \mathrm{~s}^{-1}$ cloud is in close relationship with the energetic event that originated in the Sgr A-East region. The emission tracing the $+50 \mathrm{~km} \mathrm{~s}^{-1}$ cloud extends up to $+90 \mathrm{~km} \mathrm{~s}^{-1}$.

For $\Delta l>+150^{\prime \prime}$, the $\mathrm{CO}(4-3)$ emission between $+20 \mathrm{~km} \mathrm{~s}^{-1}$ and $+90 \mathrm{~km} \mathrm{~s}^{-1}$ traces the $\mathrm{M}+0.07-0.08, \mathrm{M}+0.11-0.08$, $\mathrm{M}+0.06-0.04$, and $\mathrm{M}+0.10-0.01$ molecular clouds and gas associated with the Sickle H II region, as mentioned before. The 

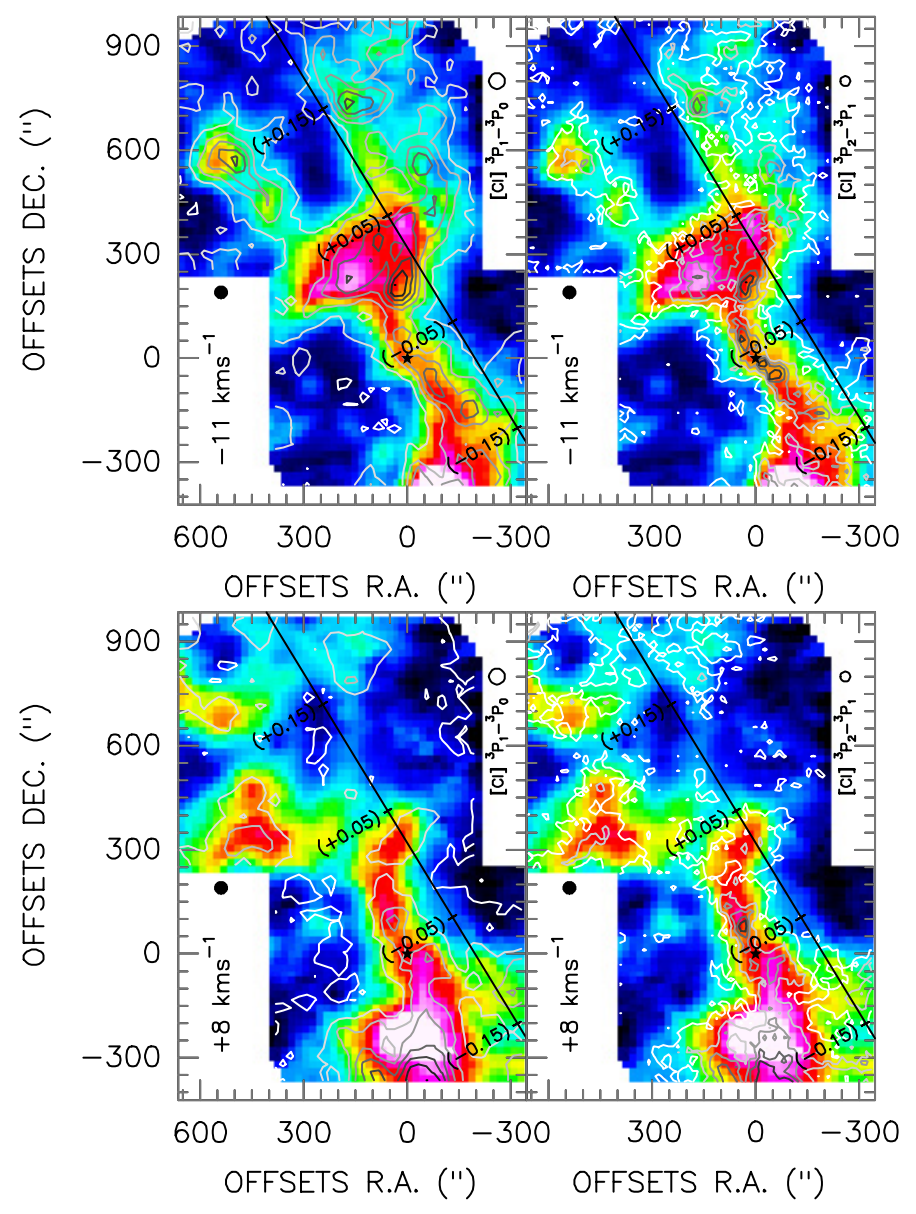

Fig. 14. Channel maps at $-11 \mathrm{~km} \mathrm{~s}^{-1}$ (top panels) and $+8 \mathrm{~km} \mathrm{~s}^{-1}$ (bottom panels) of the $\mathrm{CO}(4-3)$ (colour scale) emission, while the $[\mathrm{CI}](1-0)$ (left panels) and [CI](2-1) (right panels) lines are shown as contours. Open circles show the spatial resolution of the carbon lines, while the black filled circles show the spatial resolution of the carbon monoxide line. The $\star$ symbol shows the position of Sgr $\mathrm{A}^{\star}$. The colour scale is the same in all panels. The solid straight line shows the position of the Galactic plane at $b=0^{\circ}$; the marks denote a few Galactic longitude values for orientation.

$\mathrm{CO}(4-3)$ emission peak in this region is shifted with respect to the global $[\mathrm{CI}](1-0)$ intensity peak, around $+57 \mathrm{~km} \mathrm{~s}^{-1}$, and is comparable in magnitude to the one detected towards the $+50 \mathrm{~km} \mathrm{~s}^{-1}$ cloud, having a similar morphology to the emission below $\Delta l=+150^{\prime \prime}$ for LSR velocities between $+56 \mathrm{~km} \mathrm{~s}^{-1}$ and $+63 \mathrm{~km} \mathrm{~s}^{-1}$. This is not the case for the atomic carbon lines. The emission from the $[\mathrm{CI}](1-0)$ and $[\mathrm{CI}](2-1)$ lines above $\Delta \delta(\mathrm{J} 2000)=+250^{\prime \prime}$ is much weaker towards these molecular clouds and shows a highly asymmetric spatial distribution. Given the symmetric distribution in the $\mathrm{CO}(4-3)$ lines and the very asymmetric distribution of the $[\mathrm{CI}](1-0)$ and $[\mathrm{CI}](2-1)$ emission, it is likely that a different heating mechanism between the two regions is responsible for the gas excitation. Minh et al. (2005) attributed the enhancement of the $\mathrm{HCO}+$ emission in this region to the interaction of the gas with the shocks waves produced by the Sgr A-East supernova remnant. The [CII] emission, between $+20 \mathrm{~km} \mathrm{~s}^{-1}$ and $+90 \mathrm{~km} \mathrm{~s}^{-1}$ is much weaker than at negative LSR velocities and in general follows the distribution of the molecular gas traced by $\mathrm{CO}(4-3)$ below the Galactic plane.

Between $+20 \mathrm{~km} \mathrm{~s}^{-1}$ and $+70 \mathrm{~km} \mathrm{~s}^{-1}$, there is a prominent emission lane going from $\Delta \alpha(\mathrm{J} 2000) \sim+150^{\prime \prime}, \Delta \delta(\mathrm{J} 2000) \sim$ $+400^{\prime \prime}$ to $\Delta \alpha(\mathrm{J} 2000) \sim+400^{\prime \prime}, \Delta \delta(\mathrm{J} 2000) \sim+50^{\prime \prime}$. The feature

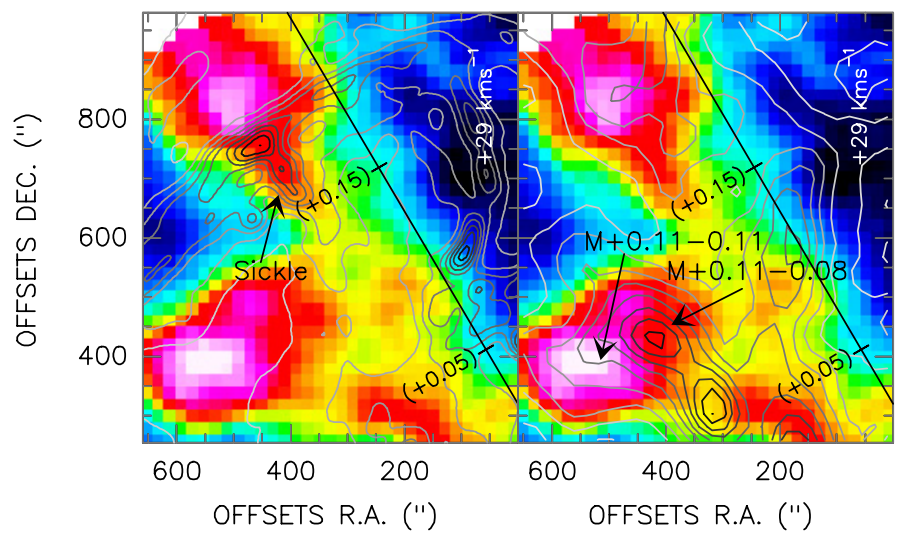

Fig. 15. Integrated intensity map $\left(3 \mathrm{~km} \mathrm{~s}^{-1}\right.$ wide) of the $\mathrm{CO}(4-3)$ emission (colour scales) centred at $+29 \mathrm{~km} \mathrm{~s}^{-1}$ with superimposed contours of the $20 \mathrm{~cm}$ continuum emission measured by Yusef-Zadeh \& Morris (1987a) showing the Sickle H II region (left panel), and $\mathrm{N}_{2} \mathrm{H}^{+}(1-0)$ contours of the integrated intensity map between -75 and $+110 \mathrm{~km} \mathrm{~s}^{-1}$ from the Mopra observations by Jones et al. (2012) showing the emission distribution of the M+0.11-0.08 cloud and the position of the M+0.11-0.11 cloud from Tsuboi et al. (1997) (right panel). The solid straight line in both panels shows the position of the Galactic plane at $b=0^{\circ}$, with marks denoting a few Galactic longitude values for orientation. The $\mathrm{CO}(4-3)$ emission related to the Sickle is located at the edge of the $\mathrm{H}$ II region while the $\mathrm{CO}(4-3)$ emission related to the $\mathrm{M}+0.11-0.11$ cloud is shifted at this LSR velocity with respect to the peak emission of the cold gas traced by diazenylium.

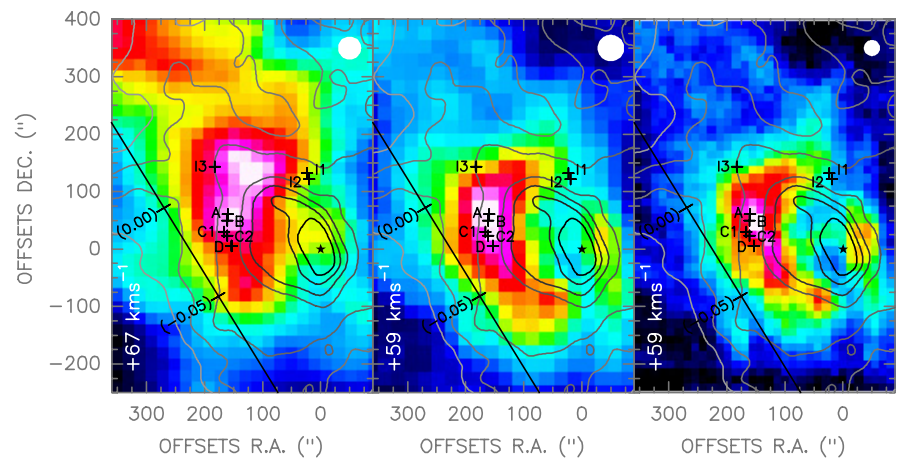

Fig. 16. Integrated intensity maps $\left(3 \mathrm{~km} \mathrm{~s}^{-1}\right.$ wide) of the $\mathrm{CO}(4-3)$, $[C I](1-0)$, and $[\mathrm{CI}](2-1)$ emission (colour scales) for the $+50 \mathrm{~km} \mathrm{~s}^{-1}$ cloud. The filled white circles represent the spatial resolution of the data, and the central LSR velocity of the maps is given in each panel. The $20 \mathrm{~cm}$ continuum emission from the $\mathrm{Sgr}$ A-East region measured by Yusef-Zadeh \& Morris (1987b) is shown as contours. The solid straight line shows the position of the Galactic plane at $b=-0.10$, with marks denoting a few Galactic longitude values for orientation. The crescentshape in all three sub-mm lines at different LSR velocities surrounding the Sgr A-East region reveals the interaction of the $+50 \mathrm{~km} \mathrm{~s}^{-1}$ cloud with the non-thermal source.

is tangent to the convex side of the $+50 \mathrm{~km} \mathrm{~s}^{-1}$ cloud. Since the [NII] emission decreases significantly for LSR velocities above $+20 \mathrm{~km} \mathrm{~s}^{-1}$, the same feature is barely detected in the [NII] line. This is shown in Fig. 17, where the [CII] and [NII] emission is overlaid on the $\mathrm{CO}(4-3)$ emission in colours. Again, the $\star$ symbol represents the position of Sgr $\mathrm{A}^{\star}$. The composite 8.0, 4.5 , and $3.6 \mu \mathrm{m}$ Spitzer/IRAC image of the Sgr A Complex in Chambers et al. (2014) shows bright emission at the same position, indicating a lower opacity than towards the positions of the bulk molecular gas. The A-I H II regions associated with the $+50 \mathrm{~km} \mathrm{~s}^{-1}$ cloud (Yusef-Zadeh \& Morris 1987a) are located 


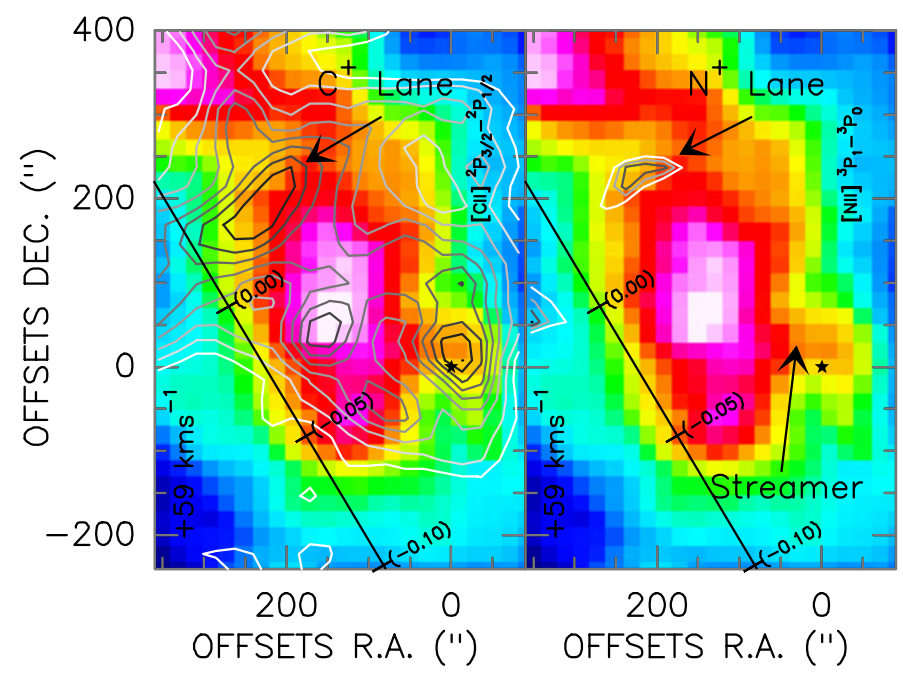

Fig. 17. Integrated intensity map $\left(3 \mathrm{~km} \mathrm{~s}^{-1}\right.$ wide) of the $\mathrm{CO}(4-3)$ (colour scale) from the $+50 \mathrm{~km} \mathrm{~s}^{-1}$ cloud centred at $+58 \mathrm{~km} \mathrm{~s}^{-1}$ with [CII] (left) and [NII] (right) emission as contours overlaid on the maps. The first contour of the high frequency lines is set at a $5 \times \sigma$ significance level, with $\sigma$ the typical noise of the map. The $\star$ symbol represents the position of $\operatorname{Sgr} A^{\star}$. The solid straight line shows the position of the Galactic plane at $b=-0^{\circ} .10$, with marks denoting a few Galactic longitude values for orientation. A prominent [CII] structure is found tangent to the convex side of the $+50 \mathrm{~km} \mathrm{~s}^{-1}$ cloud with a small [NII] counterpart.

towards the opposite side of the cloud and closer to the GC. We found no H II sources listed in the literature that correlate with the position of the emission. In general, the [NII] emission is more confined spatially than the [CII] emission, reflecting their different origin; [NII] emission is associated mainly with $\mathrm{H}$ II regions and [CII] emission tracing different stages of the ISM, including a contribution from $\mathrm{H}$ II regions.

It is interesting to note that most of the flux in the neutral atomic and molecular lines is located at Galactic latitudes below the Galactic plane $\left(b<0^{\circ}\right)$, while the flux of the ionized material traced by the $[\mathrm{NII}]$ and $[\mathrm{CII}]$ peaks above the Galactic plane, running from $\Delta \alpha(\mathrm{J} 2000)=-350^{\prime \prime}, \Delta \delta(J 2000)=-300^{\prime \prime}$ to $\Delta \alpha(\mathrm{J} 2000)=+450 ", \Delta \delta(\mathrm{J} 2000)=+1000^{\prime \prime}$ in the HerschelHIFI maps. Given the offset of the dynamical centre of the Milky Way $\left(\right.$ Sgr $\left.A^{\star}\right)$ from the centre position of the Galactic coordinate system, the location of the ionized and neutral material with respect to $b=0^{\circ}$ is not particularly meaningful, but the fact that both materials are found at different positions in the Sgr A Complex could indicate a different evolutionary stage of the gas moving along a ring-like structure (Molinari et al. 2011) whose passage through the closest point to the bottom of the gravitational potential could trigger the star formation process (Longmore et al. 2013).

\subsection{Gas streamers}

Gas streamers, first reported by Ho et al. (1991) are seen at several locations in the GC. They are thought to be the result of material from MCs (such as the $+20 \mathrm{~km} \mathrm{~s}^{-1}$ and $+50 \mathrm{~km} \mathrm{~s}^{-1}$ clouds) trapped by the central gravitational potential. They are brighter in emission from warm gas than from cold gas as they are heated on their way towards the GC (Ho et al. 1991). Using $\mathrm{NH}_{3}$ observations, Coil \& Ho (2000) reported the detection of the southern streamer stretching from the $+20 \mathrm{~km} \mathrm{~s}^{-1}$ towards the CND but not reaching the GC. No detection of the northern part of the streamer is seen in their data, probably because of the low density of the gas. Nonetheless, the southern and northern parts of the streamer are clearly traced by $\mathrm{CO}(4-3),[\mathrm{CI}](1-0)$, and $[\mathrm{CI}](2-1)$ emission in our data, as is shown in Fig. 14 at positive LSR velocities. In the $\mathrm{CO}(4-3)$ line, the northern and southern parts of the streamer appear as a single continuous structure (at least in projection) going through the CND, and are remarkably similar to the streamer shown at negative velocities, also shown in Fig. 14 (upper panels), suggesting that they could be physically related. The streamers would appear as separated entities only because of the accidental massive absorption feature at $-5 \mathrm{~km} \mathrm{~s}^{-1}$. If this were the case, it would imply that there is warm gas connecting the $-15 \mathrm{~km} \mathrm{~s}^{-1}$ cloud with the $+20 \mathrm{~km} \mathrm{~s}^{-1}$ cloud. In the same work, Coil \& Ho (2000) showed that the $+20 \mathrm{~km} \mathrm{~s}^{-1}$ and $+50 \mathrm{~km} \mathrm{~s}^{-1}$ clouds are connected by a thin molecular ridge of gas (also referred to as the eastern streamer), not seen in dust maps, and suggested that both clouds may constitute part of the same large-scale structure of gas lying along the Galactic plane. Since $\mathrm{NH}_{3}$ traces only the densest part of the gas, they fail to detect intermediate densities of the warm gas traced by $\mathrm{CO}(4-3)$. The eastern streamer is seen in $\mathrm{CO}(4-3),[\mathrm{CI}](1-0)$, and $[\mathrm{CI}](2-1)$ emission, in the velocity range $+20 \mathrm{~km} \mathrm{~s}^{-1}$ to $+50 \mathrm{~km} \mathrm{~s}^{-1}$, moving from the position of the $+20 \mathrm{~km} \mathrm{~s}^{-1}$ cloud towards the location of the $+50 \mathrm{~km} \mathrm{~s}^{-1}$ cloud surrounding the CND.

Using ${ }^{13} \mathrm{CO}(3-2)$ observations, Zylka et al. (1990) detected another streamer that is very small in spatial extent (see their Fig. 5d), reaching from the $+50 \mathrm{~km} \mathrm{~s}^{-1}$ cloud towards the CND. We detect the same feature in the $\mathrm{CO}(4-3)$ line going from $\Delta l=$ $+175^{\prime \prime}, \Delta b=-200^{\prime \prime}$ to $\Delta l=+225^{\prime \prime}, \Delta b=-100^{\prime \prime}$ between $+45 \mathrm{~km} \mathrm{~s}^{-1}$ and $+70 \mathrm{~km} \mathrm{~s}^{-1}$. The streamer is signalized in the right panel of Fig. 17, reaching towards the GC. It has a very small spatial extent and is weak in the carbon line emission. This streamer is also detected in the $1.3 \mathrm{~mm}$ dust continuum tracing free-free and thermal dust in the analysis done by Zylka et al. (1998).

A western streamer going from the $\mathrm{H}$ Region towards the $-30 \mathrm{~km} \mathrm{~s}^{-1}$ cloud and connecting with the Arched Filaments (but not going through the GC) was discussed in Sect. 3.2. The structure runs almost parallel to the Galactic plane and can be seen in the $\mathrm{CO}(4-3)$ emission between $\sim-50 \mathrm{~km} \mathrm{~s}^{-1}$ and $\sim-25 \mathrm{~km} \mathrm{~s}^{-1}$ as an almost straight line going from $\Delta l=-50^{\prime \prime}$ to $\Delta l=-400^{\prime \prime}$ at constant $\Delta b=+50^{\prime \prime}$. Atomic carbon emission is also found towards some portions of the western streamer. Since this feature does not go through the GC, it could have a different origin to the streamers previously mentioned. Nonetheless, it could reflect gas under tidal disruption orbiting the GC.

\subsection{Emission around and within the CND}

In the Herschel-HIFI maps, the CND is contained within a square of side length $100^{\prime \prime}$ centred at $\Delta \alpha(\mathrm{J} 2000)=0^{\prime \prime}$, $\Delta \delta(\mathrm{J} 2000)=0^{\prime \prime}$. This area contains the high resolution $\left(9.5^{\prime \prime}\right)$ CO(6-5) emission observed by Requena-Torres et al. (2012). In the NANTEN/SMART CO(4-3) maps measured in Galactic coordinates, this area is centred at $\Delta l \sim-202^{\prime \prime}, \Delta b \sim-165^{\prime \prime}$. Figure 18 shows the average emission within the $100^{\prime \prime} \times$ $100^{\prime \prime}$ area containing the CND. The emission extends from $-150 \mathrm{~km} \mathrm{~s}^{-1}$ to $+150 \mathrm{~km} \mathrm{~s}^{-1}$ in all lines but [NII], where the line is barely detected between $-10 \mathrm{~km} \mathrm{~s}^{-1}$ and $+30 \mathrm{~km} \mathrm{~s}^{-1}$. Overall, the $\mathrm{CO}(4-3),[\mathrm{CI}](1-0),[\mathrm{CI}](2-1)$, and $[\mathrm{CII}]$ average spectra are very similar. All present a clear emission bump at $+50 \mathrm{~km} \mathrm{~s}^{-1}$ where emission from the northern lobe of the CND dominates, while towards negative velocities, where the southern 
lobe is present, the average emission decreases also showing the strong absorption features from the loci of spiral arms along the 1.o.s. in the molecular and ionized emission.

Towards negative velocities, above $-50 \mathrm{~km} \mathrm{~s}^{-1}$, there is an excess of [CII] emission with respect to the molecular and neutral atomic emission. This excess traces a very bright $[\mathrm{CII}]$ spot towards the southern part of the CND where the peak of the integrated $\mathrm{CO}(6-5)$ intensity is also found (Requena-Torres et al. 2012). This can be clearly seen in Fig. 19 (number 1). The bright [CII] spot is detected in both atomic carbon lines and in the carbon monoxide line. In these transitions, two emission peaks are distinguishable, instead of the single emission peak in the [CII] emission. No significant [NII] emission is detected at this position. Outside the northern part of the CND, there is a second spot (number 2 in Fig. 19) at position $\Delta l \approx-75^{\prime \prime}, \Delta b \approx$ $-100^{\prime \prime}$ detected in $\mathrm{CO}(4-3)$ and in $[\mathrm{CI}](1-0)$ and $[\mathrm{CI}](2-1)$ at $\Delta \alpha(\mathrm{J} 2000) \approx+25^{\prime}, \Delta \delta(\mathrm{J} 20004) \approx+150^{\prime \prime}$. This feature shows up for the first time at around $-75 \mathrm{~km} \mathrm{~s}^{-1}$ and it is still visible up to $-55 \mathrm{~km} \mathrm{~s}^{-1}$ where the $3 \mathrm{kpc}$ absorption features appears. There is neither [NII] nor significant [CII] emission detected at this position. A third spot of emission (number 3 in Fig. 19) located just outside the northern part of the CND is detected in $\mathrm{CO}(4-3)$ at $\Delta l \approx-125^{\prime \prime}, \Delta b \approx-220^{\prime \prime}$, and barely detected in $[\mathrm{CI}](1-0)$ and $[\mathrm{CI}](2-1)$ at $\Delta \alpha(J 2000) \approx+100^{\prime \prime}, \Delta \delta(\mathrm{J} 20004) \approx$ $+25^{\prime \prime}$, traceable between $-100 \mathrm{~km} \mathrm{~s}^{-1}$ and $-55 \mathrm{~km} \mathrm{~s}^{-1}$ before it washes out with the rest of the emission. Significant [NII] or [CII] emission is not detected at this position. The different atomic carbon intensities between the second and third spots of bright $\mathrm{CO}(4-3)$ emission could be an indication of different excitation mechanisms for sources only 2 arcmin apart in the vicinity of the CND. Towards very high positive LSR velocities, a similar bright spot is found associated with the northern lobe of the CND where the $\mathrm{CO}(6-5)$ emission also shows a local maximum (Requena-Torres et al. 2012). The spot is located at $\Delta l \approx+25^{\prime \prime}, \Delta b \approx-50^{\prime \prime}$ in the $\mathrm{CO}(4-3)$ line and at $\Delta \alpha(\mathrm{J} 2000) \approx+35^{\prime \prime}, \Delta \delta(\mathrm{J} 20004) \approx+50^{\prime \prime}$ in the carbon lines. The feature is detected in all lines, except in [NII], as can be seen in the channel map at $+105 \mathrm{~km} \mathrm{~s}^{-1}$.

\subsection{High velocity gas detected in [Cl]1-0 and $\mathrm{CO}(4-3)$}

Towards the 1.o.s. to the Sgr A Complex, and at high LSR velocities $\left(\left|V_{\mathrm{lsr}}\right|>100 \mathrm{~km} \mathrm{~s}^{-1}\right)$, the gas is expected to be orbiting in the family of $\mathrm{X}_{1}$ orbits around the Galactic Centre (Jenkins \& Binney 1994). The high velocity gas (HVG) at these orbital velocities is detected in low-J CO observations, while mid-J detections are difficult owing to weak emission at those frequencies. Given the low rms noise in our submillimetre data sets, we are able to detect this gas in the $\mathrm{CO}(4-3)$ and the $[\mathrm{CI}](1-0)$ lines. At negative LSR velocities, HVG is detected between $-160 \mathrm{~km} \mathrm{~s}^{-1}$ and $-100 \mathrm{~km} \mathrm{~s}^{-1}$ at the lower right part of the $\mathrm{CO}(4-3)$ channel maps and in the lower left corner of the $[\mathrm{CI}](1-0)$ line images. This structure seems to have two velocity components along the l.o.s, judging from ${ }^{13} \mathrm{CO}(1-0)$ observations of the ongoing Mopra $\mathrm{CMZ} \mathrm{CO}(J=1-0)$ Survey (M. Burton, priv. comm.). Between $-96 \mathrm{~km} \mathrm{~s}^{-1}$ to $-73 \mathrm{~km} \mathrm{~s}^{-1}$, a remarkably comet-like structure is seen in both lines. In the $\mathrm{CO}(4-3)$ maps, it ranges from $\Delta l \sim+350^{\prime \prime}, \Delta b \sim 50^{\prime \prime}$ to $\Delta l \sim+650^{\prime \prime}, \Delta b \sim-400^{\prime \prime}$. In the $[\mathrm{CI}](1-0)$ maps, the structure spans from $\Delta \alpha(\mathrm{J} 2000)=+100^{\prime \prime}$ to $\Delta \alpha(\mathrm{J} 2000)=+550^{\prime \prime}$ at approximately constant declination $\Delta \delta(\mathrm{J} 2000)=+600^{\prime \prime}$.

At positive LSR velocities, we identified two large-scale structures with LSR velocities above $+100 \mathrm{~km} \mathrm{~s}^{-1}$. The first one can be traced between LSR velocities $+100 \mathrm{~km} \mathrm{~s}^{-1}$ to

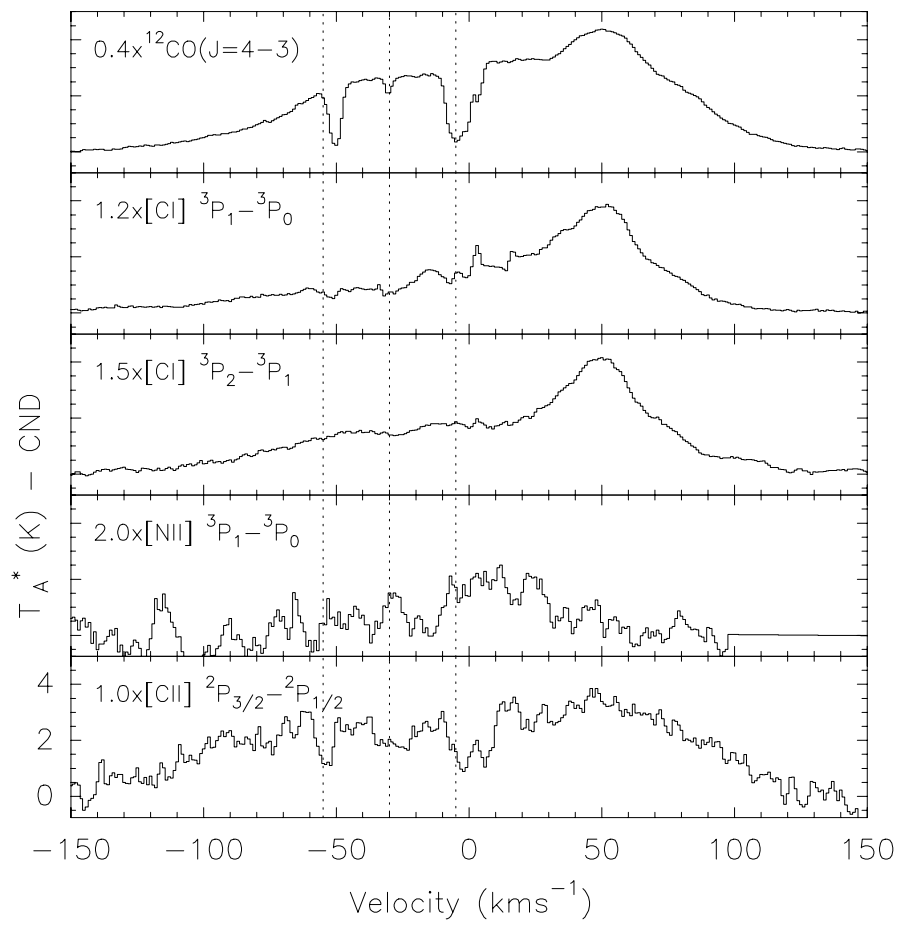

Fig. 18. Circumnuclear disk (CND) average spectra in the sub-mm/FIR lines presented in this work. The plots were created by averaging the spectra within a $100^{\prime \prime} \times 100^{\prime \prime}$ area centred at the position of Sgr $\mathrm{A}^{\star}$, from data cubes with a common $46^{\prime \prime}$ spatial resolution. Vertical dotted lines show the loci of the $3 \mathrm{kpc}, 4.5 \mathrm{kpc}$, and local arm spiral features at $\sim-55 \mathrm{~km} \mathrm{~s}^{-1}, \sim-30 \mathrm{~km} \mathrm{~s}^{-1}$, and $\sim-5 \mathrm{~km} \mathrm{~s}^{-1}$, respectively.

$+150 \mathrm{~km} \mathrm{~s}^{-1}$, and is located in the upper left and upper right regions of the $\mathrm{CO}(4-3)$ and $[\mathrm{CI}](1-0)$ channel maps, respectively. This emission was already detected in CS(2-1) by Serabyn \& Guesten (1987), but no further analysis was made. At even larger LSR velocities, between $+155 \mathrm{~km} \mathrm{~s}^{-1}$ and $+180 \mathrm{~km} \mathrm{~s}^{-1}$, a very large structure almost entirely covering the observed area is detected in both lines. Figure 20 shows the $[\mathrm{CI}](1-0)$ (colours) and $\mathrm{CO}(4-3)$ (contours) emission of this high velocity gas component at the $+175 \mathrm{~km} \mathrm{~s}^{-1}$. There is a good spatial correlation between the two lines; the carbon emission is more widespread than the carbon monoxide. From Fig. 12, the peak intensities in both lines are around $\sim 0.25 \mathrm{~K}$ in the average spectra. This structure is particularly interesting since it appears completely separated from the bulk of the gas in the $\mathrm{X}_{2}$ orbits, assumed to be at radial velocities $<+100 \mathrm{~km} \mathrm{~s}^{-1}$ in the direction of the Sgr A Complex.

In the data sets of the neutral species, we also detect the HVCC CO+0.02-0.02 previously observed by Oka et al. (2008) with high spatial resolution in $\mathrm{CO}(1-0)$ and $\mathrm{HCN}(1-0)$. The $\mathrm{HVCC}$ is clearly detected in $\mathrm{CO}(4-3),[\mathrm{CI}](1-0)$, and $[\mathrm{CI}](2-1)$ between $+100 \mathrm{~km} \mathrm{~s}^{-1}$ and $+150 \mathrm{~km} \mathrm{~s}^{-1}$. The compact source is located at the centre of the map in all observations. The [CII] detection is marginal with the emission just above the lowest contour at the $3 \sigma$ significance level, with $\sigma$ the characteristic noise of the data as summarized in Table 1.

\section{Summary}

We have presented observations of the Sgr A Complex in the sub-mm/FIR range obtained with the Herschel-HIFI satellite and NANTEN2/SMART telescope, tracing the warm molecular, atomic, and ionized gas within this region. The observations 


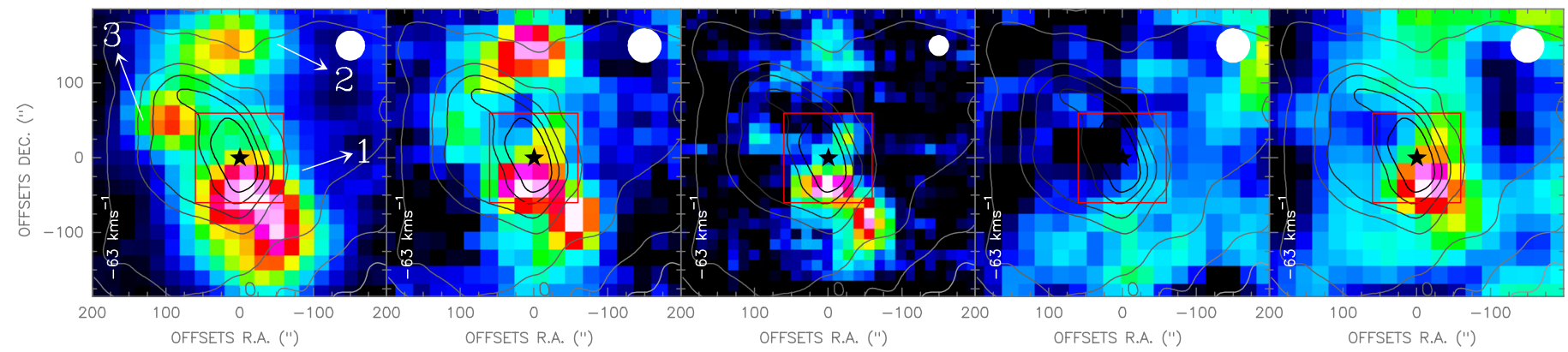

Fig. 19. From left to right: $\mathrm{CO}(4-3),[\mathrm{CI}](1-0),[\mathrm{CI}](2-1)$, [NII], and $[\mathrm{CII}]$ emission at LSR velocity $-63 \mathrm{~km} \mathrm{~s}^{-1}$ around and within the CND, denoted by the red box. The $20 \mathrm{~cm}$ continuum observations by Yusef-Zadeh \& Morris (1987a) are shown as contours. Filled white circles represent the spatial resolution in each panel. The $\star$ symbol represents the position of Sgr $\mathrm{A}^{\star}$.

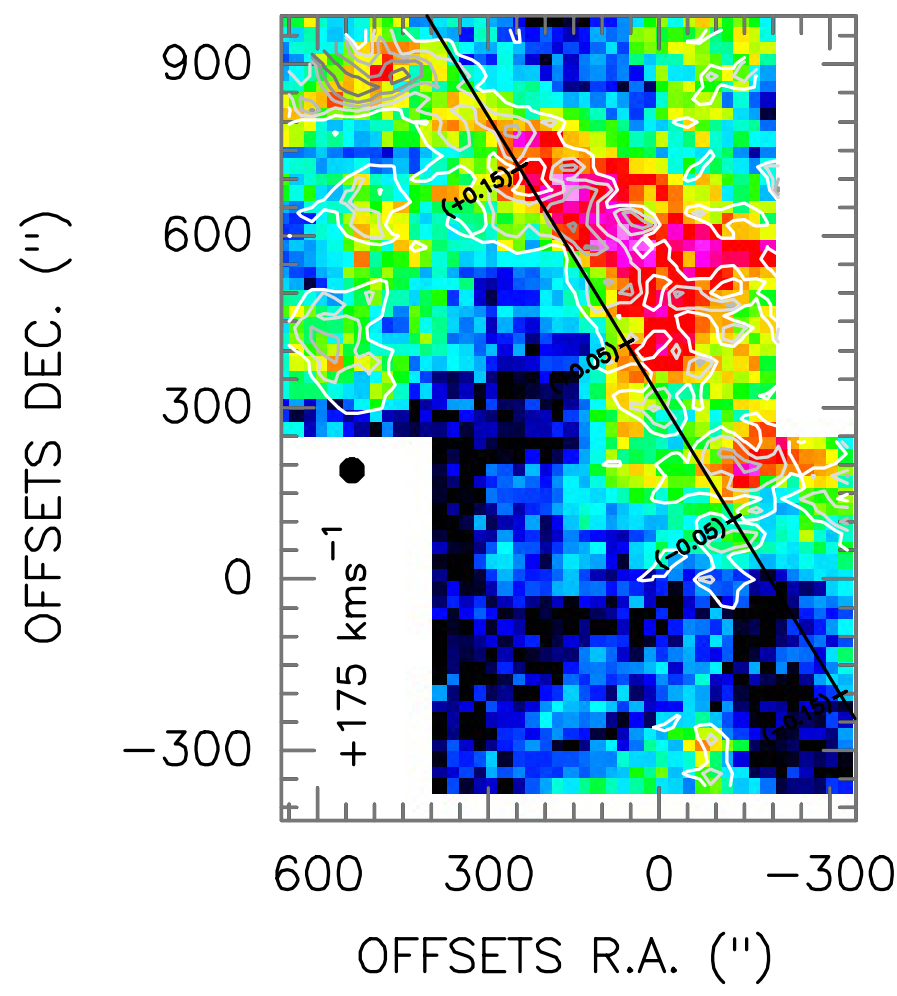

Fig. 20. Channel map of the $[\mathrm{CI}](1-0)$ (colour scale) and $\mathrm{CO}(4-3)$ (contours, black is the largest value) emission of the high velocity gas component at $+175 \mathrm{~km} \mathrm{~s}^{-1}$. The filled black circle shows the $46^{\prime \prime}$ angular resolution of the $[\mathrm{CI}](1-0)$ data. The solid straight line shows the position of the Galactic plane at $b=0^{\circ}$, with marks denoting a few Galactic longitude values for orientation. The structure belongs to the $\mathrm{X}_{1}$ orbits in the gravitational potential of the GC. The emission from both lines show large spatial coincidence, though intensity ratios can have large variations, especially towards the $\mathrm{CO}(4-3)$ peak, at the upper left corner of the image.

have high angular $\left(\leq 46^{\prime \prime}\right)$ and spectral resolution $\left(1 \mathrm{~km} \mathrm{~s}^{-1}\right)$ over a wide spectral range between $\pm 200 \mathrm{~km} \mathrm{~s}^{-1}$. We have discussed the data reduction process of the different observations and how the reference emission contamination in the Herschel-HIFI observations was recovered from the reference position measurements after standing waves were removed, and the emission was modelled and added back to the observed spectra. A cross-calibration using $[\mathrm{CI}](1-0)$ emission between the NANTEN2/SMART telescope and Herschel-HIFI satellite shows that both telescopes measure essentially the same antenna temperatures in the $T_{A}^{*}$ scale, which is a more appropriate scale for the measured intensities than the main beam temperature scale, given the large error beam pick up in both telescopes and the spatial extent of the emission in all observed lines. The main survey parameters are summarized in Table 1.

At negative LSR velocities, the Arched Filaments harbour most of the flux of the ionized gas in our observations. In the channel maps, the [CII] and [NII] emission moves from the $\mathrm{H}$ region, towards the Arched Filaments, first reaching G0.07+0.04 where the gas shows signatures of interaction with the Northern Thread, and then moving from the $\mathrm{W} 1 / \mathrm{W} 2$ to the E1/E1 filaments and to G0.10+0.02. The $[\mathrm{CI}](1-0)$ and $[\mathrm{CI}](2-1)$ emission have local maxima at the position of the $\mathrm{M}+0.04+0.03\left(-30 \mathrm{~km} \mathrm{~s}^{-1}\right.$ cloud $)$ and $\mathrm{M}+0.02-0.05\left(-15 \mathrm{~km} \mathrm{~s}^{-1}\right.$ cloud $)$, while the $\mathrm{CO}(4-3)$ peaks between the clouds. At positive LSR velocities the M2-0.13-0.08 $\left(+20 \mathrm{~km} \mathrm{~s}^{-1}\right.$ cloud) is traced by the carbon and carbon monoxide emission. The emission moves continuously with increasing LSR velocities around the CND to connect with the $\mathrm{M}-0.02-0.07$ (+50 $\mathrm{km} \mathrm{s}^{-1}$ cloud). We identify this emission lane with the eastern streamer. The $+50 \mathrm{~km} \mathrm{~s}^{-1}$ cloud is crescentshaped in the atomic carbon lines (at $\sim+59 \mathrm{~km} \mathrm{~s}^{-1}$ ) and molecular line (at $\sim+67 \mathrm{~km} \mathrm{~s}^{-1}$ ) in the $3 \mathrm{~km} \mathrm{~s}^{-1}$ wide, integrated intensity maps. This reveals the interaction of the $+50 \mathrm{~km} \mathrm{~s}^{-1}$ cloud with the Sgr A-East supernova remnant. In general, at positive LSR velocities, the distribution of the carbon emission is highly asymmetric with respect to the upper and lower parts of the Herschel-HIFI maps, separated at $\Delta \delta(\mathrm{J} 2000) \sim+250^{\prime \prime}$; most of bright emission is associated with the $+50 \mathrm{~km} \mathrm{~s}^{-1}$ cloud. On the contrary, the $\mathrm{CO}(4-3)$ emission is quite symmetric, with bright emission associated with the $+50 \mathrm{~km} \mathrm{~s}^{-1}$ cloud, but of similar intensity at the positions tracing the molecular complex including the $\mathrm{M}+0.07-0.08, \mathrm{M}+0.11-0.08, \mathrm{M}+0.06-0.04$, and $\mathrm{M}+0.10-0.01$ clouds. A local maximum in the $\mathrm{CO}(4-3)$ emission is associated with the edge of the Sickle H II region, also traced in the other sub-mm/FIR lines in our data. Emission around and within the CND show very extended emission in LSR velocity from $-150 \mathrm{~km} \mathrm{~s}^{-1}$ to $+150 \mathrm{~km} \mathrm{~s}^{-1}$ in all lines but [NII]. In addition, three emission spots at very large negative LSR velocities $\left(\sim-63 \mathrm{~km} \mathrm{~s}^{-1}\right)$ are found close to but outside (for the two northern spots) and within (for the southern spot) the CND, showing different emission strengths in our observations, which is a signature of different physical conditions. Absorption of spiral arms features along the l.o.s. are seen at many positions; the most prominent is the one at $-5 \mathrm{~km} \mathrm{~s}^{-1}$ associated with the loci of the local arm. A bright emission lane in [CII] is found associated with the convex side of the $+50 \mathrm{~km} \mathrm{~s}^{-1}$ cloud, with a very weak [NII] counterpart. Gas streamers going through the GC are clearly seen at $-11 \mathrm{~km} \mathrm{~s}^{-1}$ and $+8 \mathrm{~km} \mathrm{~s}^{-1}$ 
connecting otherwise separated entities such as the $-15 \mathrm{~km} \mathrm{~s}^{-1}$ and $+20 \mathrm{~km} \mathrm{~s}^{-1}$ clouds. The similar shape of the streamers could be an indication that they are part of the same structure, and that the lack of emission around $0 \mathrm{~km} \mathrm{~s}^{-1}$, due to spiral arm absorption along the 1.o.s., could artificially put them as separated structures. A small gas streamer going from the $+50 \mathrm{~km} \mathrm{~s}^{-1}$ cloud towards the $\mathrm{GC}$ is also detected in our $\mathrm{CO}(4-3)$ observations. A western streamer running parallel to the Galactic plane is found connecting emission associated with the $\mathrm{H}$ region with the one at the Arches Filaments. Although this streamer does not go through the GC, its elongated spatial distribution could indicate gas being tidally disrupted orbiting the GC. High velocity gas $\left(\left|V_{\mathrm{lsr}}\right|>100 \mathrm{~km} \mathrm{~s}^{-1}\right)$ structures associated with $\mathrm{X}_{1}$ orbits around the $\mathrm{GC}$ are detected in the $\mathrm{CO}(4-3)$ and $[\mathrm{CI}](1-0)$ lines. The physical conditions of this gas are expected to differ strongly from the gas at the $\mathrm{X}_{2}$ orbits (the bulk of the emission in our data sets) since gas in the outer parts of the GC is subject to strong shocks on its way towards the GC. The HVCC is also seen in $\mathrm{CO}(4-3),[\mathrm{CI}](1-0)$, and $[\mathrm{CI}](2-1)$ emission in our observations with a marginal $[\mathrm{CII}]$ detection.

Acknowledgements. This work was supported by the Comisión Nacional de Ciencia y Tecnología (CONICYT), the Deutscher Akademischer Austausch Dienst (DAAD), and the SFB956 project.

\section{References}

Abel, N. P., Ferland, G. J., Shaw, G., \& van Hoof, P. A. M. 2005, ApJS, 161, 65

Aharonian, F., Akhperjanian, A. G., Bazer-Bachi, A. R., et al. 2006, Nature, 439, 695

Allan, D. W. 1966, Proc. IEEE, 54, 221

Bally, J., Stark, A. A., Wilson, R. W., \& Henkel, C. 1987, ApJS, 65, 13

Bally, J., Aguirre, J., Battersby, C., et al. 2010, ApJ, 721, 137

Bania, T. M. 1980, ApJ, 242, 95

Caswell, J. L., Fuller, G. A., Green, J. A., et al. 2010, MNRAS, 404, 1029

Caswell, J. L., Breen, S. L., \& Ellingsen, S. P. 2011, MNRAS, 410, 1283

Chambers, E. T., Yusef-Zadeh, F., \& Ott, J. 2014, A\&A, 563, A68

Chatzopoulos, S., Fritz, T., Gerhard, O., et al. 2015, MNRAS, 447, 948

Coil, A. L., \& Ho, P. T. P. 2000, ApJ, 533, 245

Cotera, A. S., Erickson, E. F., Colgan, S. W. J., et al. 1996, ApJ, 461, 750

Dame, T. M., \& Thaddeus, P. 2008, ApJ, 683, L143

Dame, T. M., Hartmann, D., \& Thaddeus, P. 2001, ApJ, 547, 792

de Graauw, T., Helmich, F. P., Phillips, T. G., et al. 2010, A\&A, 518, L6

Figer, D. F., Rich, R. M., Kim, S. S., Morris, M., \& Serabyn, E. 2004, ApJ, 601, 319

García, P. 2015, Ph.D. Thesis, University of Cologne, Germany, http://kups . ub.uni-koeln.de/6358/

García, P., Bronfman, L., Nyman, L.-Å., Dame, T. M., \& Luna, A. 2014, ApJS, 212,2

Genzel, R., Stacey, G. J., Harris, A. I., et al. 1990, ApJ, 356, 160

Goldsmith, P. F., Li, D., Bergin, E. A., et al. 2002, ApJ, 576, 814

Graf, U. U., Heyminck, S., Michael, E. A., et al. 2003, Proc. SPIE, 4855, 322

Guan, X., Stutzki, J., Graf, U. U., et al. 2012, A\&A, 542, L4

Guesten, R., Walmsley, C. M., \& Pauls, T. 1981, A\&A, 103, 197

Higgins, D. 2011, Ph.D. Thesis, National University of Ireland, Maynooth

Ho, P. T. P., Ho, L. C., Szczepanski, J. C., Jackson, J. M., \& Armstrong, J. T. 1991, Nature, 350, 309

Horn, J., Siebertz, O., Schmülling, F., et al. 1999, Exp. Astron., 9, 17

Hunter, S. D., Bertsch, D. L., Catelli, J. R., et al. 1997, ApJ, 481, 205
Jenkins, A., \& Binney, J. 1994, MNRAS, 270, 703

Jones, P. A., Burton, M. G., Cunningham, M. R., et al. 2012, MNRAS, 419, 2961

Jones, P. A., Burton, M. G., Cunningham, M. R., Tothill, N. F. H., \& Walsh, A. J. 2013, MNRAS, 433, 221

Klein, B., Hochgürtel, S., Krämer, I., et al. 2012, A\&A, 542, L3

Kramer, C., 1997, Calibration of spectral line data at the IRAM $30 \mathrm{~m}$ radio telescope, Version 2.1, January 24th

Lang, C. C., Goss, W. M., \& Wood, D. O. S. 1997, ApJ, 474, 275

Lang, C. C., Figer, D. F., Goss, W. M., \& Morris, M. 1999a, AJ, 118, 2327

Lang, C. C., Morris, M., \& Echevarria, L. 1999b, ApJ, 526, 727

Lang, C. C., Goss, W. M., \& Morris, M. 2001, AJ, 121, 2681

Lang, C. C., Goss, W. M., \& Morris, M. 2002, AJ, 124, 2677

Lang, C. C., Goss, W. M., Cyganowski, C., \& Clubb, K. I. 2010, ApJS, 191, 275

Law, C. J., Yusef-Zadeh, F., \& Cotton, W. D. 2008, ApJS, 177, 515

Linke, R. A., Stark, A. A., \& Frerking, M. A. 1981, ApJ, 243, 147

Longmore, S. N., Kruijssen, J. M. D., Bally, J., et al. 2013, MNRAS, 433, L15

Nagayama, T., Omodaka, T., Handa, T., et al. 2007, PASJ, 59, 869

Martin, C. L., Walsh, W. M., Xiao, K., et al. 2004, ApJS, 150, 239

Mezger, P. G., Zylka, R., Salter, C. J., et al. 1989, A\&A, 209, 337

Mezger, P. G., Duschl, W. J., \& Zylka, R. 1996, A\&ARv, 7, 289

Minh, Y. C., Kim, S.-J., Pak, S., et al. 2005, New Astron., 10, 425

Mizutani, K., Maihara, T., Matsuhara, H., et al. 1994, ApJS, 91, 613

Molinari, S., Bally, J., Noriega-Crespo, A., et al. 2011, ApJ, 735, L33

Müller, M., \& Jellema, W. 2014, The HIFI Beam: Release \#1, Release Note for Astronomers, 19 Sept.

Oka, T., Hasegawa, T., Sato, F., Tsuboi, M., \& Miyazaki, A. 1998, ApJS, 118, 455

Oka, T., Nagai, M., Kamegai, K., Tanaka, K., \& Kuboi, N. 2007, PASJ, 59, 15

Oka, T., Hasegawa, T., White, G. J., et al. 2008, PASJ, 60, 429

Oka, T., Onodera, Y., Nagai, M., et al. 2012, ApJS, 201, 14

Ossenkopf, V. 2008, A\&A, 479, 915

Ossenkopf, V., Röllig, M., Neufeld, D. A., et al. 2013, A\&A, 550, A57

Ott, S. 2010, Astronomical Data Analysis Software and Systems XIX, 434, 139

Ott, J., Weiß, A., Staveley-Smith, L., Henkel, C., \& Meier, D. S. 2014, ApJ, 785, 55

Pardo, J. R., Cernicharo, J., \& Serabyn, E., 2001, IEEE Trans. on Antennas and Propagation, 49/12, 1683

Pierce-Price, D., Richer, J. S., Greaves, J. S., et al. 2000, ApJ, 545, L121

Pineda, J. L., Mizuno, N., Stutzki, J., et al. 2008, A\&A, 482, 197

Pineda, J. L., Mizuno, N., Röllig, M., et al. 2012, A\&A, 544, A84

Poglitsch, A., Stacey, G. J., Geis, N., et al. 1991, ApJ, 374, L33

Porquet, D., Rodriguez, J., Corbel, S., et al. 2003, A\&A, 406, 299

Reid, M. J., Menten, K. M., Brunthaler, A., et al. 2014, ApJ, 783, 130

Requena-Torres, M. A., Güsten, R., Weiß, A., et al. 2012, A\&A, 542, L21

Roelfsema, P. R., Helmich, F. P., Teyssier, D., et al. 2012, A\&A, 537, A17

Sawada, T., Hasegawa, T., Handa, T., et al. 2001, ApJS, 136, 189

Schuller, F., Menten, K. M., Contreras, Y., et al. 2009, A\&A, 504, 415

Serabyn, E., \& Guesten, R. 1987, A\&A, 184, 133

Sidoli, L., Belloni, T., \& Mereghetti, S. 2001, A\&A, 368, 835

Simon, R., Graf, U., Kramer, C., Stutzki, J., \& Onishi, T. 2007, Report on beam efficiency measurements at NANTEN after surface adjustment, Version of February 13th

Stark, A. A., \& Bania, T. M. 1986, ApJ, 306, L17

Stolovy, S., Ramirez, S., Arendt, R. G., et al. 2006, J. Phys. Conf. Ser., 54, 176

Tanaka, K., Oka, T., Matsumura, S., Nagai, M., \& Kamegai, K. 2011, ApJ, 743, L39

Timmermann, R., Genzel, R., Poglitsch, A., et al. 1996, ApJ, 466, 242

Tsuboi, M., Ukita, N., \& Handa, T. 1997, ApJ, 481, 263

Watson, M. G., Willingale, R., Hertz, P., \& Grindlay, J. E. 1981, ApJ, 250, 142

Yusef-Zadeh, F., \& Morris, M. 1987a, ApJ, 320, 545

Yusef-Zadeh, F., \& Morris, M. 1987b, AJ, 94, 1178

Yusef-Zadeh, F., Morris, M., \& Chance, D. 1984, Nature, 310, 557

Zhao, J.-H., Desai, K., Goss, W. M., \& Yusef-Zadeh, F. 1993, ApJ, 418, 235

Zylka, R., Mezger, P. G., \& Wink, J. E. 1990, A\&A, 234, 133

Zylka, R., Philipp, S., Duschl, W. J., et al. 1998, The Central Regions of the Galaxy and Galaxies, Proc. IAU Symp., 184, 291 


\section{Appendix A: Baseline distortions in the [NII] and [CII] Lines}

The baseline distortions of the $[\mathrm{NII}]$ and $[\mathrm{CII}]$ observations carried out with the HEBs mixers of the Herschel-HIFI satellite are shown in Figs. A.1 and A.2. The baseline distortion in Fig. A.1 is produced by the combination of the diplexer coupling at this frequency, the electrical standing waves in the HEB mixers, and the IF frequency dependence sensitivity of the HEB mixers, which are more sensitive to lower IF frequencies. The red line in the figure denotes the LSR velocity range in which the average antenna temperature was calculated, while the dashed black line shows the result of the average. In Fig. A.2 and average OTF line of the [CII] observations illustrates the electrical standing wave patter present in the vertical polarization data produced by an impedance mismatch between the mixer and the first low noise amplifier (LNA) due to a too high LO power pumping (Higgins 2011).

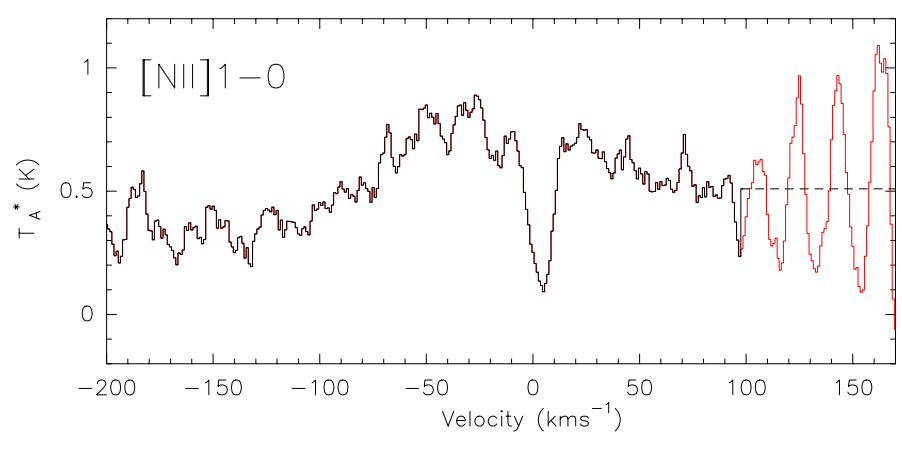

Fig. A.1. [NII] average spectrum of the $\mathrm{V}$ polarization observations over the whole map. The red line indicates the distortion of the baseline between $+94 \mathrm{~km} \mathrm{~s}^{-1}$ and $+170 \mathrm{~km} \mathrm{~s}^{-1}$ present in all the spectra. The dashed line over the same LSR velocity interval shows the result of the method utilized in getting rid of the distortion by replacing the measured antenna temperatures by their average value within the same LSR velocity interval.

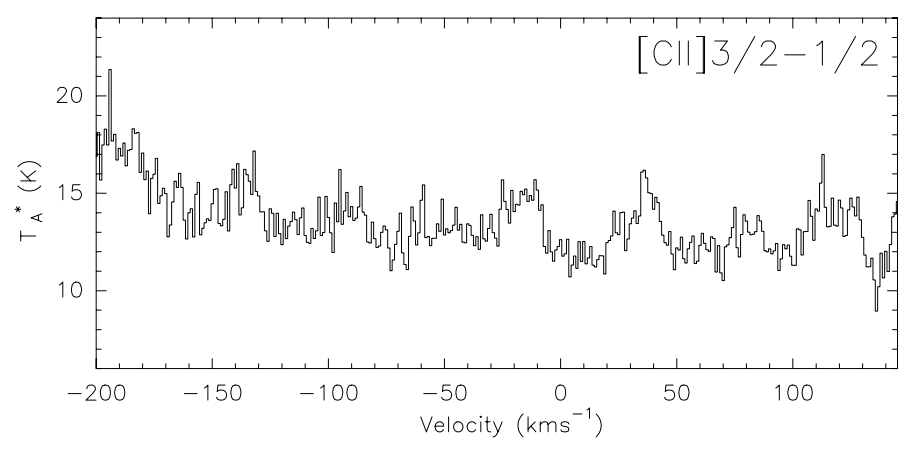

Fig. A.2. $[\mathrm{CII}]$ average OTF line of the V polarization spectra. The curvature of the average spectrum together with the distortion of the baseline across the bandpass are the product of an overpump of the $\mathrm{V}$ polarization mixer due to excessive LO power (Higgins 2011). Such spectra were filtered out by setting the $T_{\text {A,rms }}^{*}$ threshold to $2.3 \mathrm{~K}$. 